\title{
Novel Fabrication Approach of a Porous Silicon Biocompatible Membrane Evaluated within a Alveolar Coculture Model
}

Marcus A. C. Williams ${ }^{\mathrm{a}}$, Cooper Wiens ${ }^{\mathrm{a}}$, Adam Hamilton ${ }^{\mathrm{a}}$, Sophie Mancha ${ }^{\mathrm{b}}$, Madeline Stalder ${ }^{\mathrm{b}}$, Koby Vargas ${ }^{\mathrm{b}}$, Jerry Crawford ${ }^{\mathrm{b}}$, Yiyan Li ${ }^{\mathrm{b}}$, Sarah Schreiner ${ }^{\mathrm{c}, \mathrm{d}}$, David J. Blake ${ }^{\mathrm{e}}$, and Jeffrey R. Jessing $^{\mathrm{b}}$ *

a. Fort Lewis College, Department of Chemistry and Biochemistry, Durango, CO

b. Fort Lewis College, Department of Physics and Engineering, Durango, CO

c. STROBE NSF Science and Technology Center

d. JILA, University of Colorado Boulder, Boulder, CO

e. Fort Lewis College, Department of Biology, Durango, CO

*Denotes corresponding author

Tel. +9702476742

E-mail address: jrjessing@fortlewis.edu 


\begin{abstract}
The use of conventional in vitro and preclinical animal models often fail to properly recapitulate the complex nature of human diseases and hamper the success of translational therapies in humans [1-3] Consequently, research has moved towards organ-on-chip technology to better mimic human tissue interfaces and organ functionality. Herein, we describe a novel approach for the fabrication of a biocompatible membrane made of porous silicon (PSi) for use in organ-on-chip technology that provides key advantages when modeling complex tissue interfaces seen in vivo. By combining well-established methods in the semiconductor industry with organ-on-chip technology, we have developed a novel way of producing thin $(25 \mu \mathrm{m})$ freestanding PSi biocompatible membranes with both nano ( $\sim 15.5 \mathrm{~nm}$ diameter pores) and macroporous ( $\sim 0.5 \mu \mathrm{m}$ diameter pores) structures. To validate the proposed novel membrane, we chose to recapitulate the dynamic environment of the alveolar blood gas exchange interface in alveolar co-culture. Viability assays and immunofluorescence imaging indicate that human pulmonary cells remain viable on the PSi membrane during long-term culture (14 days). Interestingly, it was observed that macrophages can significantly remodel and degrade the PSi membrane substrate in culture. This degradation will allow for more intimate physiological cellular contact between cells, mimicking a true blood-gas exchange interface as observed in vivo. Broadly, we believe that this novel PSi membrane may be used in more complex organ-on-chip and lab-on-chip model systems to accurately recapitulate human anatomy and physiology to provide further insight into human disease pathology and preclinical response to therapeutics.
\end{abstract}

Keywords: Lung-on-a-chip, porous silicon, bio-membrane, co-culture, through-etching 


\section{Graphical Abstract}

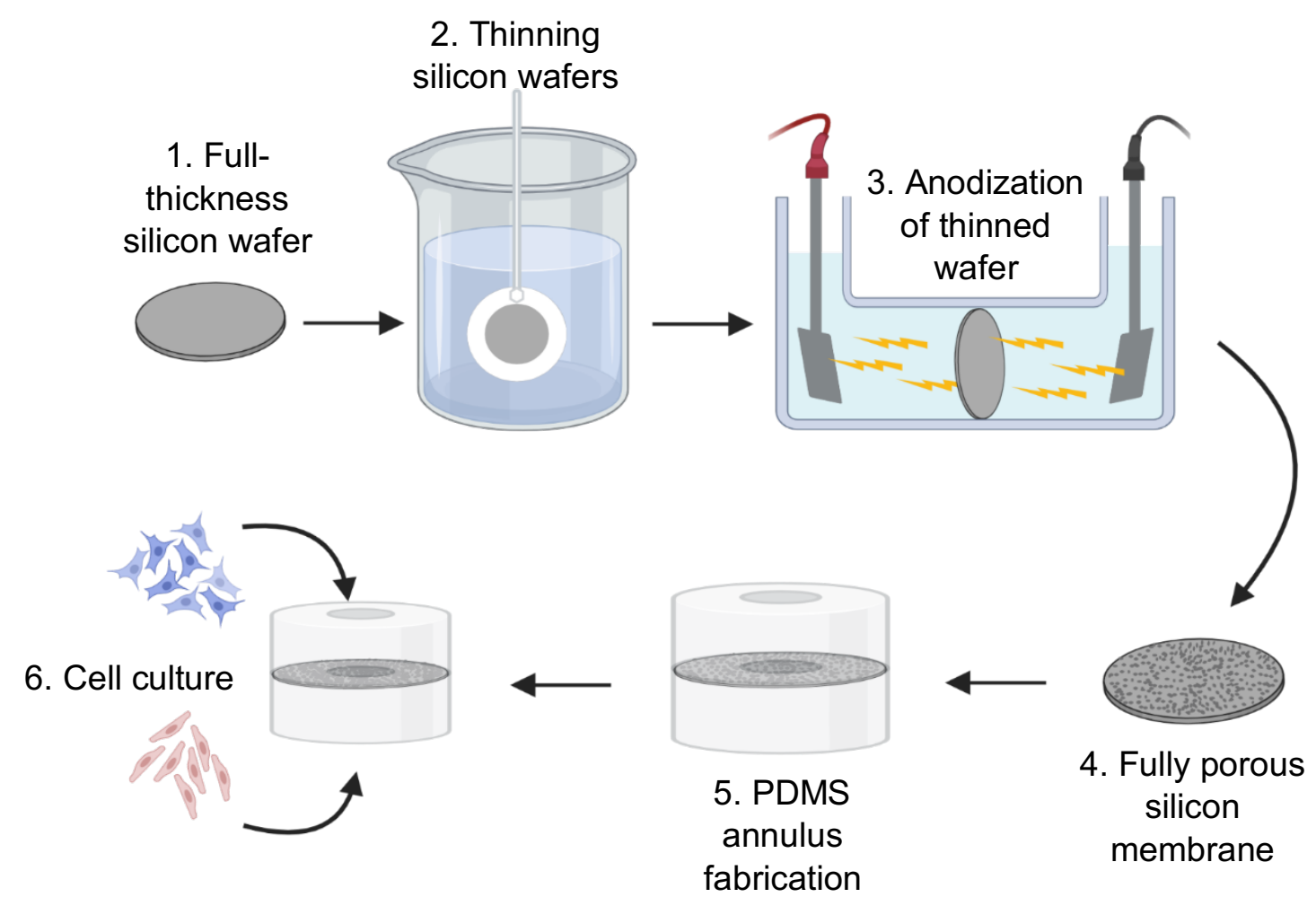

\section{Introduction}

In the scope of biomedical research many advances have been made in the past few decades that have been pivotal to our understanding of disease pathology, treatment, and pre-clinical drug therapy response. Many of these discoveries thus far have been made with the use of multiple animal models and traditional cell culture experimentation [4-7]. While these methods are useful when studying single cellular pathways or overarching biological processes, they often falter when studying the meticulous nature of human disease and limit our abilities to develop effective therapeutics [8-10]. It has become increasingly clear that conventional animal models and cell culture do not provide the necessary information to extrapolate human biology and fail to recapitulate human anatomy and physiology [11-13]. Therefore, there is a great need for advanced model systems that can more accurately facilitate intercellular communication and tissue architecture to model human disease progression and that can predict the efficacy of future treatment options. 
In order to address this gap in knowledge, researchers have developed organ-on-chip devices or tissue chips [14]. Organ-on-chip devices are in vitro models that incorporate multiple cell types and act to recapitulate the anatomy and physiology within human organ systems. By utilizing techniques pioneered in the microchip manufacturing industry, biomedical engineers can create micro-fabricated engineered systems that can be used to culture living cells [1]. These organ-onchip devices can maintain healthy cell populations with lineage-specific function as observed in human organ systems. Through controlling the micro-environment in which the cell populations reside, cell behavior is remarkably similar to cell populations in living tissue [1,15-18]. Current studies to date have utilized these organ-on-chip devices to study human skin, bone, cartilage, kidney, liver, blood vessels, intestine, lung, and multiple other organ systems [19-26], and have recently been used to rapidly identify antiviral therapies [27].

Conventional organ-on-chip devices are often constructed of polydimethylsiloxane (PDMS), a flexible, biocompatible polymer that serves as the scaffold for in vitro microfluidic organ systems. The first seminal lung-on-a-chip (LOAC) device published by Huh et al. follows this convention $[15,16]$ an other LOAC studies have utlized thin PDMS membranes that can incorporate cyclic strain thereby mimicing the biomechanical forces observed in alveolar tissue [29]. Within these devices, an engineered porous PDMS membrane is located at the interface between two or more different cell types that allow for the transfer of nutrient media, gasses, and signaling molecules. For example, the utility of PDMS has been exploited to create airways on a chip exposed to inhaled toxicants, such as cigarette smoke [30]. While this design is reliable and easily fine-tuned, there are numerous technical challenges involved in making an extremely thin PDMS barrier $(<10 \mu \mathrm{m})$ [28,29]. In addition, the hydrophobicity of PDMS leads to absorption and binding of hydrophobic molecules and requires additional treatments of exogenous extracellular matrix (ECM) components for cell adhesion, making this material sub-optimal for cell culture [31-33]. Newer second generation LOAC devices have recently been created replacing the PDMS material with stretchable membranes made of collagen and elastin to better emulate the three-dimensional alveolar structure [34]. Other groups have created improved artificial basement membranes to separate different cell types through a combination of lithography and vacuum assisted UV curing techniques by electrospun gelatin nanofibers [35]. To this end, we sought to evaluate a new material substrate (porous silicon) for structural membranes based on a bottom-up fabrication 
technique that allow for cell adherence along with exhibiting properties of biocompatibility and mechanical flexibility.

For this new material substrate, we examined the extent to which crystalline silicon could be used as an alternate structural material in lieu of PDMS membranes. Due to its popularity in the semiconductor industry, natural abundance, cost-effectiveness and process maturity, silicon was an ideal material to pursue [28,36]. Large areas of crystalline silicon can be thinned to less than 2 micrometers $(\mu \mathrm{m})$, which dramatically increases its mechanical flexibility [37]. One particular form of silicon, termed porous silicon (PSi), has been shown to exhibit many unique beneficial properties including flexibility, biocompatibility, hydrophilicity, optical transparency, and biodegradability [38-42]. The structure of PSi can be modified to create micro- and nano-sized pores [25]. In addition, PSi membranes have also been shown to have excellent optical transparency which facilitates imaging with fluorescence microscopy [42]. These properties, in conjunction with the existing methods utilized to favorably alter the material, further validated the use of silicon as a structural membrane material in an organ-on-chip device. Ultra-thin macroporous silicon (macro-PSi) and nanoporous (nano-PSi) silicon membranes have been previously created by a lift-off method which ultimately proved to have limited reproducibility and mechanical stability [43]. In this work we introduce a novel method for fabricating and implementing ultra-thin fully PSi membranes into our alveolar co-culture system. To further validate the potential for these PSi membranes in organ-on-chip technology and to advance this in vitro platform, novel LOAC fabricated devices were cultured with human pulmonary epithelial, endothelial, and macrophage cells in long-term culture to more accurately mimic the parenchymal, vascular, and immune compartments of the lung.

\section{Methods and Materials}

\subsection{Overview}

Two-inch polished silicon wafers were thinned to approximately 2 - $25 \mu \mathrm{m}$ via anisotropic etching in a strong aqueous solution of potassium hydroxide $(\mathrm{KOH})$. The thinned wafers were anodized in an electrolyte solution of hydrofluoric acid to produce either macropores or nanopores. Using widely used methods to produce nanopores, thinned wafers were anodized to obtain thinned membranes with pores penetrating throughout the entirety of the membrane. The thinned, porous membranes were bonded between two identical toroidal pieces of PDMS to create an annulus with 
two cell culture wells separated by the PSi membrane. Multiple cell types were plated onto the porous membrane within the culture well, and cellular viability, lineage, and interactions were characterized utilizing multiple microscopy methods. Once cellular interactions were evaluated, the porous membranes were integrated into a prototype microfluidic device to determine their potential use in organ-on-chip devices. The microfluidic device utilized SU-8 photoresist patterning, producing a mold used to create microchannels within two pieces of PDMS, which were then aligned and bonded on either side of a PSi membrane to create the device prototype.

\subsection{Potassium hydroxide wafer thinning}

In a $1000 \mathrm{~mL}$ beaker, $600 \mathrm{~g}$ reagent-grade potassium hydroxide $(\mathrm{KOH})$ purchased from SigmaAldrich (St. Louis, MO) was dissolved in $600 \mathrm{~mL}$ of deionized water (50 wt $\%$ ). The $\mathrm{KOH}$ solution was suspended in an isothermal water bath held at $90^{\circ} \mathrm{C}$ as shown in Figure $2 \mathrm{C}$. A two-inch double-sided polished, p-type (boron-doped), ( $\left.\begin{array}{lll}1 & 0 & 0\end{array}\right)$ orientation silicon wafer with a resistivity of 0.01-0.1 $\Omega \cdot \mathrm{cm}$ purchased from University Wafer (Boston, MA) was mounted in custom-fabricated polytetrafluoroethylene (PTFE) etch rings and was anisotropically etched in the $\mathrm{KOH}$ solution to a thickness between 2 - $25 \mu \mathrm{m}$ determined by SEM analysis of cross-sections. The etch rates were determined experimentally for each wafer throughout the process.

\subsection{Electrochemical anodization to obtain nanopores}

The thinned wafer was mounted in a dual-tank custom-made polyvinylchloride (PVC) anodization cell as shown in Figure $3 \mathrm{~A}$ and B. Both tanks were filled with electrolyte solution consisting of 1.5-parts $48 \mathrm{wt} \%$ hydrofluoric acid (HF) in $\mathrm{H}_{2} \mathrm{O}$ to 1-part reagent-grade 200 proof ethanol purchased from Sigma-Aldrich (St. Louis, MO) (1.5:1-HF:EtOH by volume). One square inch platinum electrodes within the cell were connected to a power source (Keithley $24101100 \mathrm{~V}$ Source Measurement Unit), and a current density of $90 \mathrm{~mA} / \mathrm{cm}^{2}$ was applied to induce electrochemical anodization of the thinned silicon wafer. Utilizing calculated anodization rates $(\sim 1$ $\mu \mathrm{m} / \mathrm{min}$ ), wafers were anodized to $150 \%$ of the calculated "through-anodization" time (37.5 minutes), to create thin though-etched porous membranes. Through-anodization was confirmed utilizing scanning electron microscopy and MATLAB image analysis. Percent porosity and average pore diameter of nanopores were calculated utilizing scanning electron microscopy and MATLAB image analysis. 


\subsection{Electrochemical anodization to obtain macropores}

The thinned wafer was mounted in a dual-tank custom-made PTFE anodization cell. Both tanks were filled with $48 \mathrm{wt} \% \mathrm{HF}$ ( $4 \%$ by wt) in reagent-grade dimethylformamide purchased from Sigma-Aldrich (St. Louis, MO). One square inch platinum electrodes within the cell were connected to a power source (Keithley 2410 1100V Source Measurement Unit), and a current density of $4 \mathrm{~mA} / \mathrm{cm}^{2}$ was applied to induce electrochemical anodization of the thinned silicon wafer. These anodization parameters resulted in an etch rate of approximately $3.3 \mu \mathrm{m} / \mathrm{hour}$. Percent porosity and average pore diameter of macropores were calculated via scanning electron microscopy and MATLAB image analysis.

\subsection{PDMS formation}

SylgardTM 184 was mixed with SylgardTM Silicone Elastomer Curing Agent (10\% wt) and loaded into a $10 \mathrm{~mL}$ BD-Syringe (Luer-Lok tip) and degassed under vacuum. From the resulting PDMS, $0.5 \mathrm{~mL}$ was extruded into each individual well within a Falcon ${ }^{\circledR}$ Multi-Well 24-well cell culture tray and cured at $65^{\circ} \mathrm{C}$ for 1 hour. Cylindrical holes $(5 \mathrm{~mm}$ diameter) were created in the center of the resulting molded PDMS discs with a biopsy punch to achieve a small annulus as shown in Figure 5. Small sections of the fully porous membrane were then sandwiched between two aligned annuli, and subsequently bonded using an ozone generator (Electro-Technic Products Inc. model BD-20) and heated at $80^{\circ} \mathrm{C}$ for 10 minutes on a hotplate to further bond the PDMS.

\subsection{SU-8 PDMS microfluidic channel molding and fabrication}

A clean silicon wafer was statically coated with $\sim 1 \mathrm{~mL}$ of SU-8 2050 negative photoresist purchased from Kayaku Advanced Materials (Westborough, MA) and spun 1,500 rpm for 1 minute. The wafer was then subsequently soft-baked in a convection oven at $65^{\circ} \mathrm{C}$ for 10 minutes and then at $95^{\circ} \mathrm{C}$ for 50 minutes which gave a layer of SU-8 2050 that was approximately $125 \mu \mathrm{m}$ thick. Following this, the wafer was covered with a photomask designed in AutoCAD and exposed to a UV light source for 4 minutes. As seen in Figure S3, the mask was designed to have circular inlets and outlets $(3.14 \mathrm{~mm} 3)$ that connect to a circular center culture well $(4.91 \mathrm{~mm} 3)$ via rectangular fluidic channels $(1 \mathrm{~mm} \times 0.5 \mathrm{~mm})$. After exposure, the wafer was once again softbaked at $65^{\circ} \mathrm{C}$ for 10 minutes and $95^{\circ} \mathrm{C}$ for 50 minutes. The wafer was then submerged in SU-8 developer purchased from Kayaku Advanced Materials (Westborough, MA) for 4 minutes and was 
then rinsed with reagent-grade isopropyl alcohol purchased from Sigma-Aldrich (St. Louis, MO), to produce on the wafer a negative-pattern mold for PDMS microchannel fabrication. The mold was then hard-baked in a convection oven at $250^{\circ} \mathrm{C}$ for 10 minutes. Once the negative-pattern mold cooled to room temperature, the wafer was placed in a $70 \mathrm{~mL}$ aluminum weighing dish purchased from Fisher Scientific (Hampton, NH).

\subsection{PDMS microfluidic device formation}

8.5 mL of PDMS was extruded over the negative pattern mold in the aluminum weighing dish and set to de-gas under vacuum for 20 minutes. After, the weighing dish and contents were baked in a convection oven at $65^{\circ} \mathrm{C}$ for 1 hour and set to cool overnight. Once cured overnight, the patterned PDMS was then extracted and layered with a PSi membrane in the center culture well and aligned and bonded.

\subsection{Scanning electron microscopy}

All scanning electron microscopy analysis for process development/verification and post-culture characterization was performed using a JEOL JSM-7100F Field Emission Scanning Electron Microscope.

\subsection{Confocal microscopy}

Multi-spectral confocal microscopy with a Leica TCS-SP8 outfitted with spectral hybrid detectors was performed on PSi nanoporous membranes ( $25 \mu \mathrm{m}$ thickness) that were mounted with ProLong Gold and \#1.5 high precision coverslips. A series of confocal spectral images with emission bandwidths steps of $20 \mathrm{~nm}$ was acquired with LASX software in xyl mode from 500-660 nm on a single detector during simultaneous excitation at the $488 \mathrm{~nm}$ and $594 \mathrm{~nm}$ wavelengths. The respective laser power settings were used to reproduce experimental conditions used in the indirect immunofluorescence staining. All images were exported in tiff format and spectral data are expressed as mean gray level intensity.

\subsection{Biological reagents}

Calcein-AM, Alexa Fluor ${ }^{\mathrm{TM}} 594$ Phalloidin and Prolong Gold mounting media were obtained from ThermoFisher Scientific (Waltham, MA). Mouse monoclonal anti-occludin antibodies were obtained from Sigma Aldrich (St. Louis, MO). Rabbit polyclonal anti-VE-cadherin antibodies 
(rabbit IgG) were obtained from Cell Signaling Technology (Danvers, MA). Alexa 488conjugated goat anti-rabbit antibodies and Alexa 549-conjugated goat anti-mouse antibodies were obtained from ThermoFisher Scientific (Waltham, MA).

\subsection{Cell culture and seeding}

H441 cells (human lung epithelial cell line) and THP-1 cells (human macrophages) were purchased from the American Type Culture Collection (Manassas, VA). Both H441 and THP-1 cells were cultured at $37^{\circ} \mathrm{C}$ in a $5 \% \mathrm{CO}_{2}$ incubator in RPMI- 1640 medium containing $10 \%$ fetal bovine serum (FBS) and antibiotics (Life Technologies, Grand Island, NY). THP-1 cells were not differentiated with phorbol-12-myristate-13-acetate (PMA) prior to seeding. Human lung microvascular endothelial cells (HMVECs) were purchased from Lonza (Walkersville, MD). Cells were cultured at $37^{\circ} \mathrm{C}$ in a $5 \% \mathrm{CO}_{2}$ incubator in EGMTM-2 MV Microvascular Endothelial Cell Growth Medium-2 BulletKitTM (Lonza). Cells were maintained and passaged as recommended by the manufacturer. The H441 epithelial cells were fed every 2 to 3 days and subcultured using $0.25 \%$ trypsin (Invitrogen) to detach the adherent cells. The THP-1 macrophages were also fed every two to three days and remained suspension monocyte cells at a concentration of less than $1 \times 10^{6}$ cells $/ \mathrm{mL}$. The HMVECs were passaged according to the manufacturer's specifications using the recommended subculturing reagents for these cells including Trypsin/EDTA (CC-5012), Trypsin Neutralizing Solution (CC-5002), and HEPES Buffered Saline Solution (CC-5022). HMVECs had growth medium changed every 48 hours.

\subsection{Fluorescence microscopy to determine cell adherence, long-term viability, and co-culture on} nanoporous silicon

Viable H441 cells and HMVECs were trypsinized, counted, and adhered to thinned PSi membranes at a density of 120 cells $/ \mu \mathrm{L}$ of media. To allow for cell adherence to the PSi substrate, cells were incubated at $37^{\circ} \mathrm{C}$ in a $5 \% \mathrm{CO}_{2}$ incubator overnight. Cells were fixed with $4 \%$ paraformaldehyde, permeabilized with TX-100, blocked in 5\% BSA buffer, and then stained with phalloidin and mounted with mounting media containing DAPI. Cells were visualized with a BX60 Olympus epifluorescence microscope with an Olympus camera. To determine the long-term viability of H441 cells and HMVECs, cells were counted and plated as described above. After 14 days of culture, cells were stained with $4 \mu \mathrm{g} / \mathrm{mL}$ Calcein AM in PBS and incubated for 1 hour at $37^{\circ} \mathrm{C}$. Calcein $\mathrm{AM}$ is a cell-permeant dye that is used to determine cell viability in eukaryotic cells. 
In viable cells, the nonfluorescent Calcein AM is converted to green fluorescent Calcein, after acetoxymethyl ester hydrolysis by intracellular esterases. Viable cells were visualized with an Olympus IX50 inverted microscope.

To determine whether cells can successfully adhere to both sides of the nano-PSi membrane, H441 cells were seeded to one side of the PSi membrane for 24 hours. The next day HMVECs were seeded on the opposite side of the membrane. Cells were then fixed, permeabilized and blocked as described above. Cells were then stained with the correct working dilution of anti-occludin and anti-VE cadherin antibodies simultaneously in PBS containing 3\% FBS and incubated at $4{ }^{\circ} \mathrm{C}$ overnight. The membranes were then washed, and the respective fluorescent secondary antibodies were incubated with the membranes at room temperature for 2 hours prior to washing and mounting with mounting medium. Human epithelial and endothelial cells were visualized with a BX60 Olympus epifluorescence microscope with an Olympus camera as described above.

\subsection{Scanning electron microscopy of cellular populations to visualize cell-membrane interactions}

Cells were fixed in 4\% paraformaldehyde for 1 hour on a rocker. Following fixation, the cells were washed with PBS ( $2 \times 4$ min). The cells were then treated with 1\% osmium tetroxide in PBS for 30 minutes and then rinsed twice with deionized $\mathrm{H} 2 \mathrm{O}$. The cells were then washed in increasing concentrations of EtOH for 5 minutes at each concentration $(25 \%, 50 \%, 75 \%, 95 \%)$. Next, the cells were washed with $100 \%$ EtOH for 5 minutes two times $(2 \times 5$ min). Following EtOH washes, the cells were treated with reagent grade hexamethyldisilazane purchased from Sigma-Aldrich (St. Louis, MO) for 10 minutes twice $(2 \times 10 \mathrm{~min})$. Following washes, the PSi membranes were extracted from the annuli cell culture wells, dried with nitrogen, and sputter-coated with $\mathrm{Au} / \mathrm{Pd}$ at 75 millitorrs with a current of 45 milliamps for 30 seconds using a Denton Vacuum Desk II. The resultant sample was then imaged in an SEM.

\subsection{Statistical Analysis}

Data are presented as mean \pm standard error of the mean (SEM). Analyses were performed using GraphPad Prism 6.0 (San Diego, CA). First, Levene's test for homogeneity of variance was performed with the complete PSi thickness data (two biological replicates with three to five technical replicates $(n=3-5))$. A difference between the variances was observed, therefore, a nonparametric test (Kruskal-Wallis) was performed. Significance was noted at $\mathrm{P}<0.05$. 


\section{Results and Discussion}

\subsection{Porous Silicon Membrane Fabrication, Characterization, and Implementation}

In this work, we have developed methods for obtaining novel ultra-thin biological PSi membranes for use in organ-on-chip devices. The combination of thinning a silicon wafer using $\mathrm{KOH}$ followed by the electrochemical anodization of the thinned wafer provides a simple and reproducible method of creating thin, fully porous membranes. The basic process parameters were characterized and optimized yielding a tailored membrane with respect to thickness, pore size, and porosity. Adjusting these parameters, we serendipitously designed the PSi membrane to allow diffusion of small gas molecules, small signaling proteins, and to facilitate the trafficking of immunological cells as observed in vivo. Furthermore, we utilize these novel PSi membranes in surrogate models for the blood-gas exchange interface observed within the human alveolus (Figure 1). In order to do so, we evaluated the viability of cell types observed at this interface on each side of the novel PSi membrane, both independently, and in co-culture where cells are cultured on both sides of the membrane within their respective regions. To the best of our knowledge, this novel PSi membrane exhibits many unique properties that have yet to be studied and exploited in organ-on-chip systems.

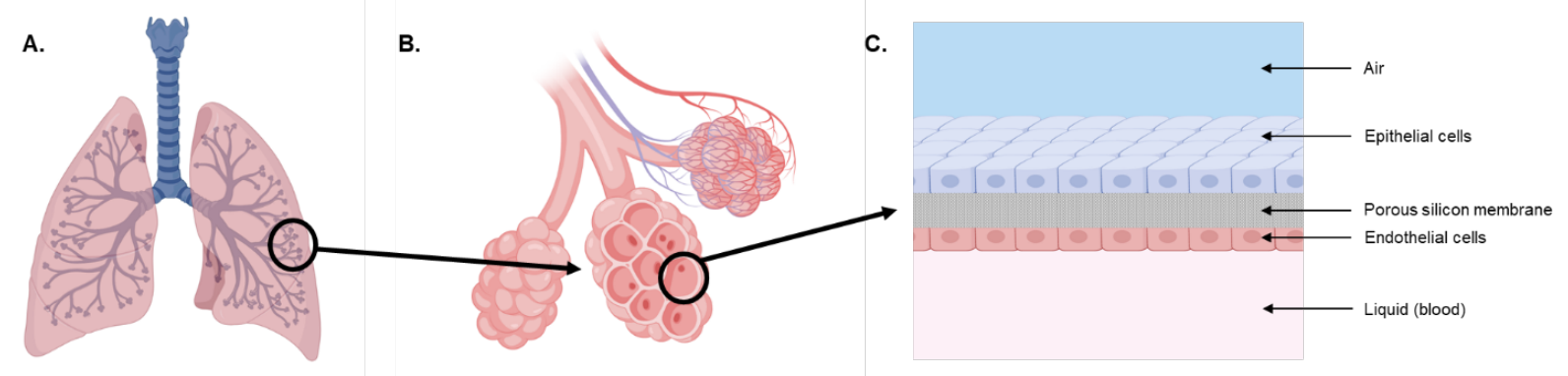

Figure 1. Figure 1: Human alveolar lung structure is accurately modeled by the novel PSi membrane Lung-on-a-chip (LOAC) microfluidic device. (A) Schematic of human lung with branching bronchi. (B) Schematic of human alveoli at the distal ends of branching bronchi at the blood-gas exchange interface. (C) A representation of an engineered LOAC device showing the nanostructured PSi membrane adjacent to both human epithelial and endothelial cells mimicking the parenchymal and vascular compartments of the lung.

To mimic the anatomical distance between endothelial cell membranes (vasculature) and pulmonary epithelial cell membranes (parenchyma) at the basement membrane interface of an alveolus $(\sim 1 \mu \mathrm{m})[42,43]$, we developed methods for producing uniform thinned bulk silicon 
membranes using double-sided polished (DSP) silicon wafers via anisotropic wet chemical etching (Figure 2). After recording initial thickness measurements (typically on the order of $280 \mu \mathrm{m}$ ), wafers were mounted in PTFE rings which left both sides of the wafer exposed and then submerged in an apparatus containing a super-saturated solution $(\sim 17.8 \mathrm{M}, 50 \mathrm{wt} \%)$ of $\mathrm{KOH}$.
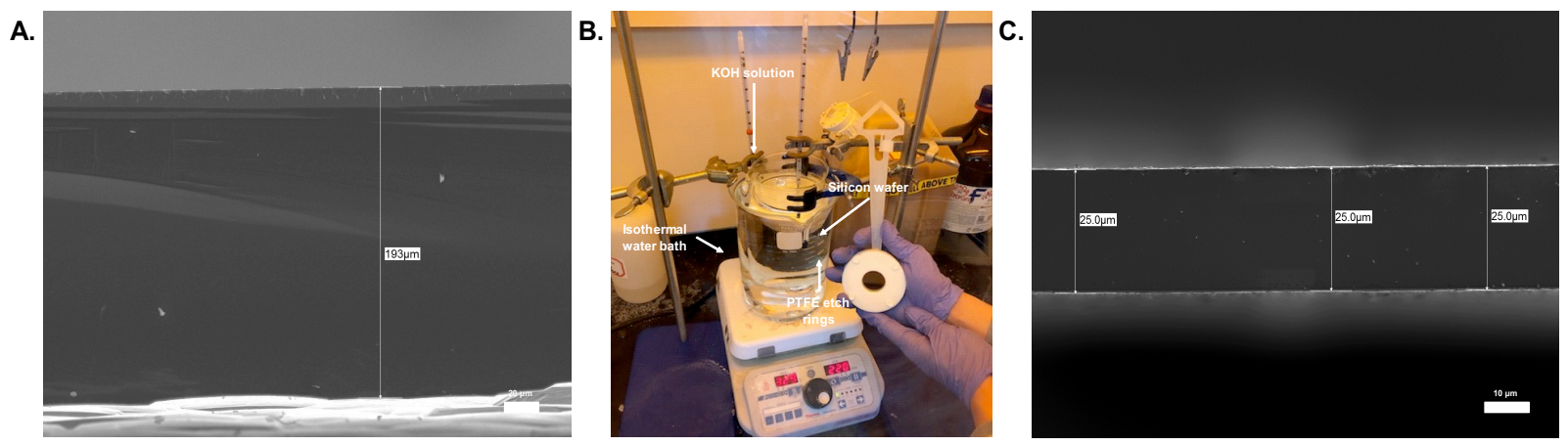

Figure 2. Wet chemical etching apparatus allows for uniform thinning of double-sided polished (DSP) silicon wafers (A) Scanning electron microscopy (SEM) image of DSP silicon wafer prior to chemical etching measured at $193 \mu \mathrm{m}$ in width. (B) DSP silicon wafer mounted in polytetrafluoroethylene (PTFE) "etch rings" prior to being submerged in wet chemical etching apparatus containing super-saturated solution of potassium hydroxide $(\sim 17.8 \mathrm{M}, 50 \mathrm{wt} \%)$. (C) SEM cross-sectional thickness image of silicon wafers post-wet chemical etching measured at 25.0 $\mu \mathrm{m}$.

While submerged, the bulk silicon was anisotropically etched enabling uniform thinning of the silicon wafer bilaterally. Following one hour of wet chemical etching, wafers were re-measured and etch rates were determined for each individual silicon wafer. Bilateral anisotropic etch rates of the wafers used varied between $\sim 1.5 \mu \mathrm{m} / \mathrm{min}$ and $\sim 2.5 \mu \mathrm{m} / \mathrm{min}$. This methodology yields the ability to precisely control the resultant thickness of the silicon membranes. As a result, we were able to achieve consistently thin wafers $(25 \mu \mathrm{m})$. (Figure $2 \mathrm{C}$ )

Through-wafer electrochemical anodization of the thinned silicon substrates yielded permeable silicon membranes with the formation of tunable nano- to macropores (Figure 3). In order to electrochemically etch the wafers, thinned silicon wafers were placed into a dual-tank etch cell with a hydrofluoric acid electrolyte solution introduced on both sides (Figure 3 A and B). Once the etch cell was secured, a current was applied to the system, which resulted in the dissolution of silicon in localized regions directly correlating to porous structures. Electrochemical anodization parameters were optimized to allow for the formation of porous structures that penetrated 
throughout the thinned silicon wafers at a uniform rate. Experiments were conducted with a current density of $90 \mathrm{~mA} / \mathrm{cm}^{2}$ in a solution of $\mathrm{HF}$ and ethanol EtOH for periods of time calculated such that nanopores, with an average value of $15.5 \mathrm{~nm} \pm 6.21 \mathrm{~nm}$ in diameter, spanned the entire thickness of the membrane (Figure $3 \mathrm{C}$ and D, Figure S1 B). To further explore possible silicon membrane types with varying pore sizes, silicon wafers were etched with a current density of 4 $\mathrm{mA} / \mathrm{cm}^{2}$ in solutions of HF and dimethylformamide which yielded macropore structures with an average pore diameter of $0.469 \mu \mathrm{m} \pm 0.210 \mu \mathrm{m}$ (Figure $3 \mathrm{E}$ and F, Figure S1 D). Following anodization, the thinned wafers were analyzed with scanning electron microscopy (SEM) to determine electrochemical etch rates for each process. Additionally, SEM images were used to observe the morphological characteristics of the pore structure and quantify percent porosity of both macro-PSi ( $\sim 76.8 \%$ porous) and nano-PSi ( $\sim 50.7 \%$ porous) (Figure $\mathrm{S} 1)$. The formation of macropore structures $(\sim 3.3 \mu \mathrm{m} /$ hour $)$ was markedly slower in comparison to etch rates associated with the formation of nanopore structures $(\sim 1 \mu \mathrm{m} / \mathrm{min})$. As a result, the remainder of the study utilized parameters developed to obtain nanoporous membranes that could be fabricated in a shorter time frame for the sake of feasibility. Utilizing estimated electrochemical etch rates of the silicon wafers and known thicknesses, thinned wafers were etched to $150 \%$ completion to achieve relatively uniform "through-etching" (Figure 4). Through-etching greatly increases the surface area of silicon exposed to atmosphere and is vulnerable to native oxide growth. To limit oxide growth on the membranes and maintain relative uniformity between all samples, the membranes were stored under high vacuum at $<10^{-6}$ Torr. 
A.

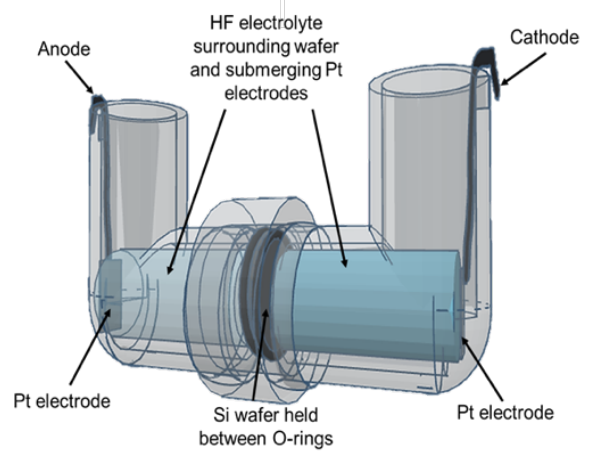

c.

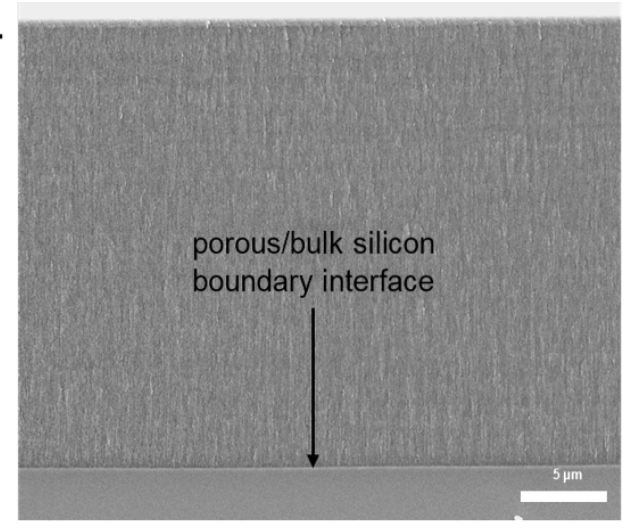

E.

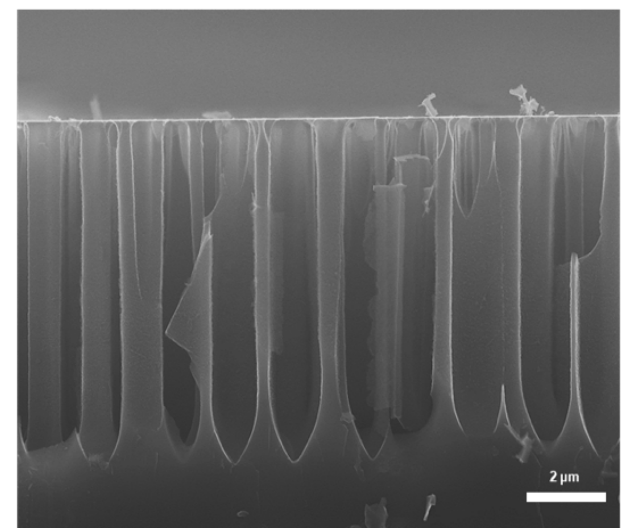

B.

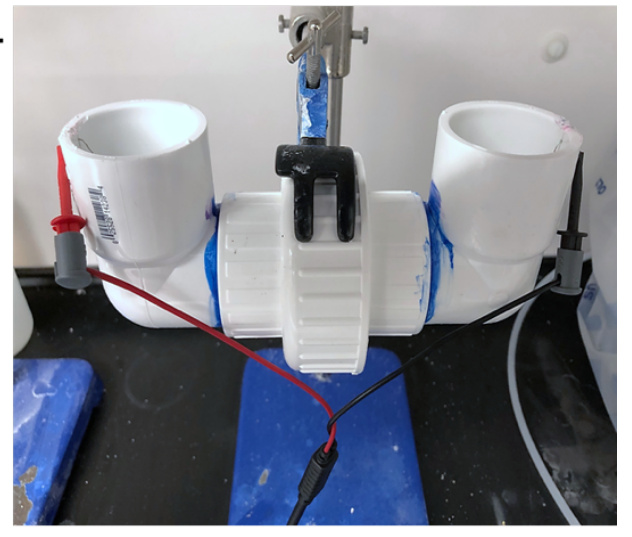

D.

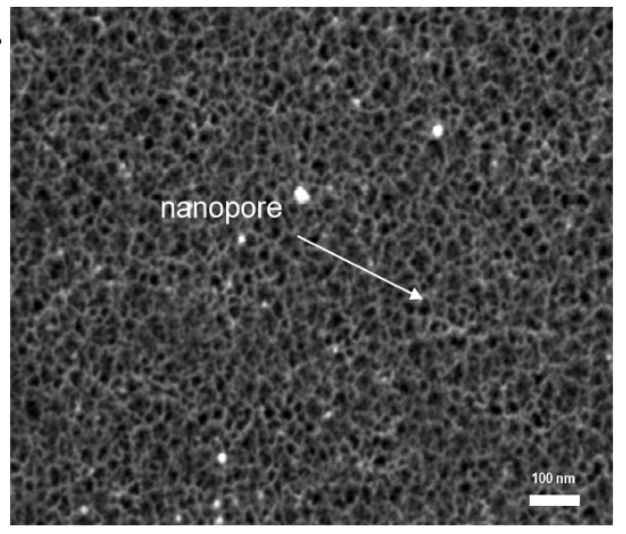

F.

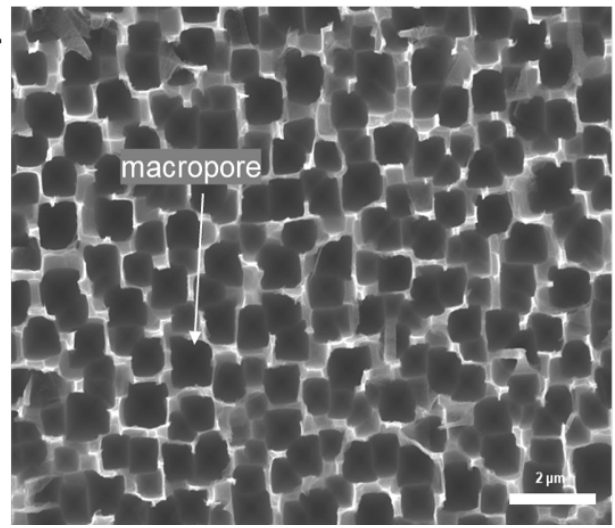

Figure 3. Successful thinned silicon wafers mounted in a custom-made double-tank electrochemical etching cell to create nano- and macro-PSi membranes. (A) Schematic of the etch cell used for electrochemical etching of thinned silicon to afford PSi membranes. (B) PTFE etch cell used for electrochemical anodization. (C) SEM cross-sectional image of a silicon wafer demonstrating successful nanopore propagation. (D) SEM top-down image of nanopore structures giving a porous surface with a percent porosity of $50.7 \%$ and an average pore diameter of $15.5 \mathrm{~nm}$ $\pm 6.21 \mathrm{~nm}$. (E) SEM cross-sectional image of a silicon wafer demonstrating macropore propagation throughout the silicon membrane. (F) SEM top-down image of macropore structures giving a porous surface with a percent porosity of $76.8 \%$ and an average pore diameter of 0.469 $\mu \mathrm{m} \pm 0.210 \mu \mathrm{m}$. 
A.

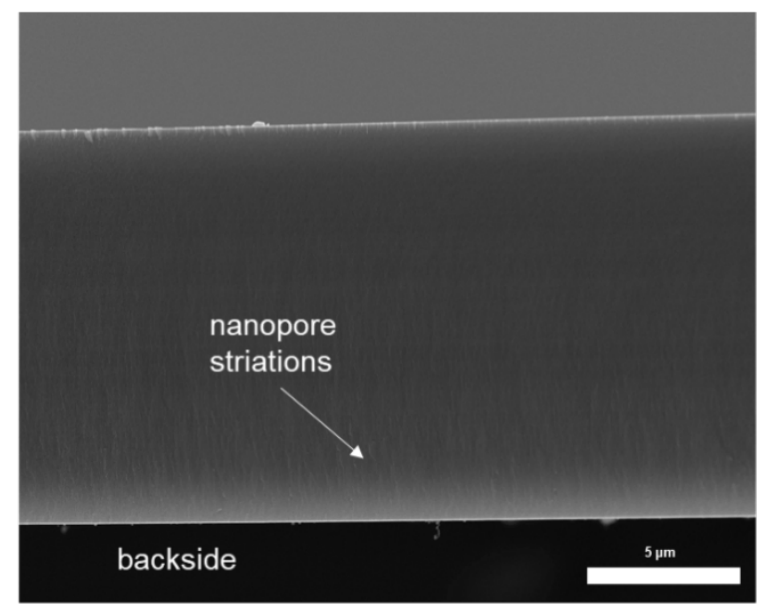

B.

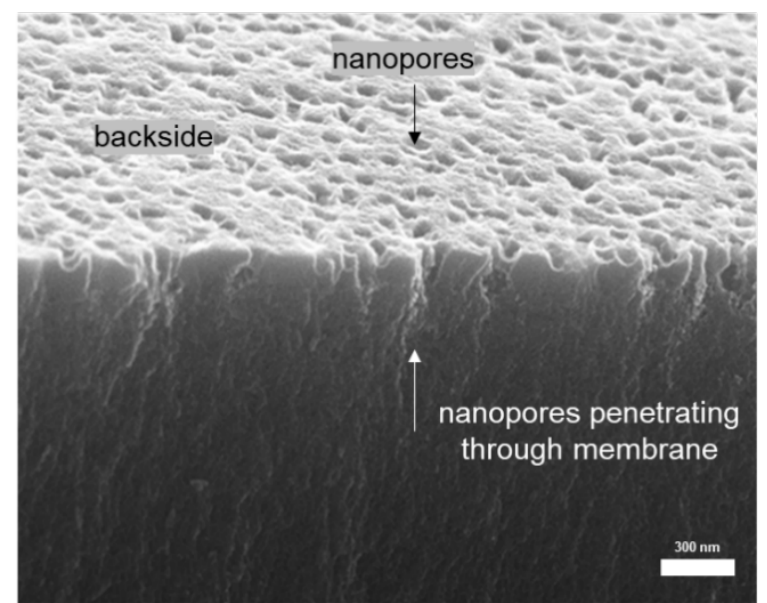

c.

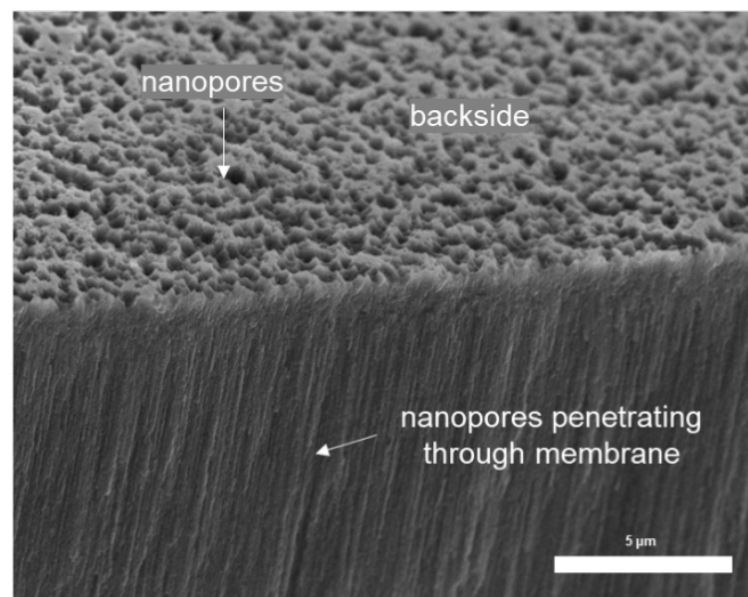

Figure 4. Silicon wafers anodized to $150 \%$ completion contain nanopores permeating throughout the entirety of the membrane to afford fully PSi membranes. (A) Cross section of a fully anodized silicon wafer where pore striations can be observed spanning the entirety of the wafer imaged at 2,500x. (B and C) Angled images of the "backside" of two different PSi membranes where nanopores are seen to be punching through confirming the PSi membranes are fully porous imaged at 43,000x and 6,000x respectively.

To replicate a 3D organ-on-a-chip system, a PDMS annulus was used as a housing unit to encapsulate the PSi membrane (Figure 5). The PSi membrane is suspended between two PDMS outer layers which serves two purposes. First, cells plated in suspension have bilateral fluid transfer as seen in conventional organ-on-chip technologies [46]. Second, multiple cell types could be cultured (epithelial and endothelial) with the membrane separating the cell types, acting as the interstitial space as found in in vivo human lungs. 

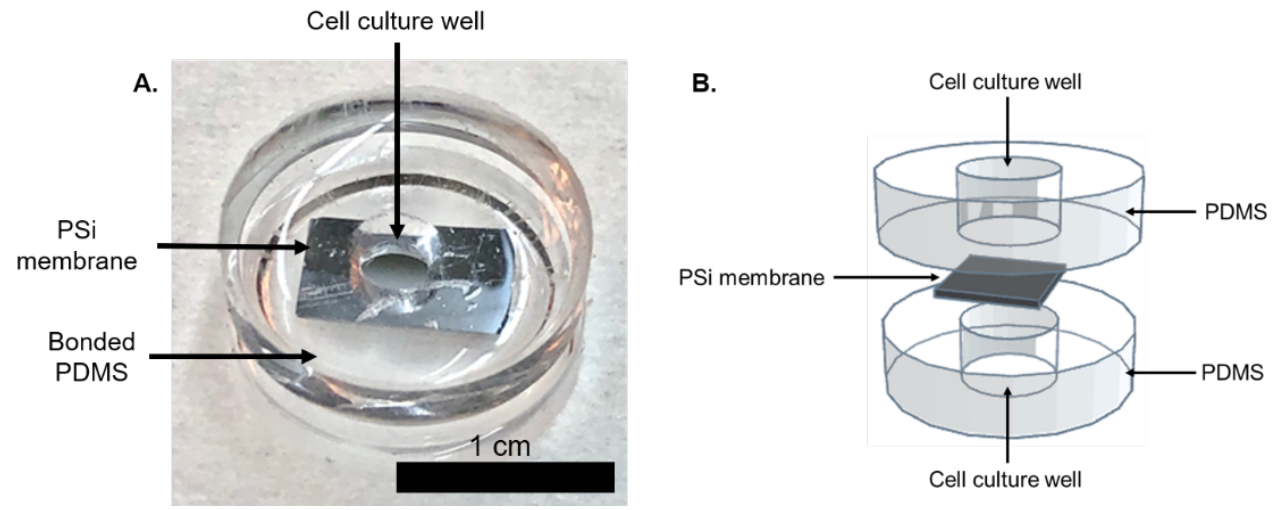

Figure 5. Tissue culture annulus allows for cells to be cultured in suspension on the PSi membrane. (A) Image of PSi membrane incorporated between two PDMS annuli via ozone bonding. (B) Schematic diagram detailing tissue culture annulus layering and design.

\subsection{Spectral Characterization of PSi membranes}

The PSi membranes exhibited a very small ( $<11$ gray levels) multispectral autofluorescence characteristic in the yellow-orange spectral range (570 to 600) when excited with both the 488 and 594 lasers. Multispectral autofluorescence was absent in the specific emission ranges collected for AF488 (505 nm-535 nm) and AF594 (620 nm-650 nm), suggesting that the use of these fluorophores will permit for confocal microscopy free from PSi autofluorescence artifact.

\subsection{Cell adherence, viability, and morphology}

In order to study the cellular interactions of epithelium and endothelium at the alveolocapillary barrier, a well-established pulmonary co-culture model was used including human distal lung epithelial cells (H441 cells) and human microvascular endothelial cells (HMVECs) [45]. This model has been used to validate previously developed seminal LOAC devices $[16,17]$. To this end, H441 cells and HMVECs were initially plated on PSi membranes to determine whether these materials were suitable to maintain cell viability and cell adherence. Importantly, no additional surface treatments, which are characteristic of previous organ-on-chip devices such as collagen and fibronectin coating or oxygen plasma treatments were required $[16,40,48]$. Cells were plated on PSi and then visualized with phalloidin and 4',6-diamidino-2-phenylindole (DAPI) to determine cell morphology and adherence. H441 cells and HMVECs exhibited normal cell morphology associated with viable cells (round homogeneously stained nuclei and polymerized actin filaments 
at the cell periphery) and adhered to PSi structures (Figure $6 \mathrm{~A}$ and B). These results are in accordance with previous studies indicating surface roughness enhances cell adherence due to increased surface area [48].

Longer-term cell culture viability was also assessed to ensure that cellular growth and proliferation on the PSi membrane was possible for future, extended ( $>14$ days in culture) cell culture experiments. For these studies, H441 cells, HMVECs, and human macrophages (THP-1 cells) were all plated individually on the PSi membranes for up to 14 days. After 14 days in culture, adherent epithelial and endothelial cells were viable, metabolically active, and had normal cell morphology as evidenced by the cellular staining with Calcein-AM (Figure $6 \mathrm{C}$ ). Cell adherence and morphology of adherent human macrophages over this period was also noted and examined by SEM analysis. These data indicate that thinned PSi membranes provide a cell appropriate, nontoxic, supportive and adherent surface to maintain cellular co-cultures with at least three separate cell types.

Next, we determined whether H441 cells and HMVECs could adhere to opposite sides of a PSi membrane which had been previously thinned to 25 microns, to successfully create a co-culture system. H441 cells were plated and allowed to adhere overnight. After 24 hours, HMVECs were seeded on the opposite side of the PSi membrane and allowed to adhere overnight. The membrane was then fixed and stained with immunofluorescent antibodies to identify the individual cell types; human epithelial cells that preferentially express Occludin and human endothelial cells that preferentially express Cadherin 5 [50]. Each cell type adhered to their respective (opposing) sides of the thinned, PSi membrane (Figure $6 \mathrm{D}$ and $\mathrm{E}$ ) and could be uniquely identified. Cell adherence and normal cell morphology were also confirmed through SEM analysis (Figure $6 \mathrm{~F}$ and $\mathrm{G}$ ). During immunofluorescence visualization, the HMVECs were the first cells to be observed as they were closest to the objective lens. More importantly, the H441 cells could be visualized through the thinned PSi membrane on the opposite side of the membrane, indicating that the PSi membrane was thin enough and of a compatible material to image both sides in studies requiring more than one fluorescent molecule such as DAPI, AF488 and AF594 (Figure 6 D and E). These results support the tenet that using optically transparent materials such as thin PSi membranes with pore 
diameters in the nanometer range decreases undesired optical effects which impede bilateral microscopy [51].

A.

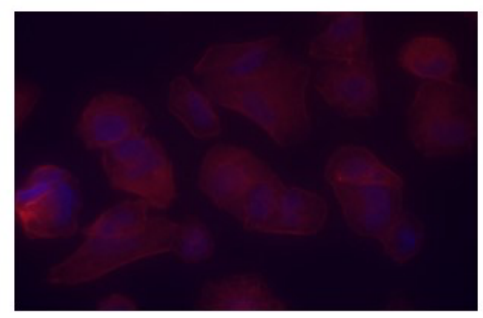

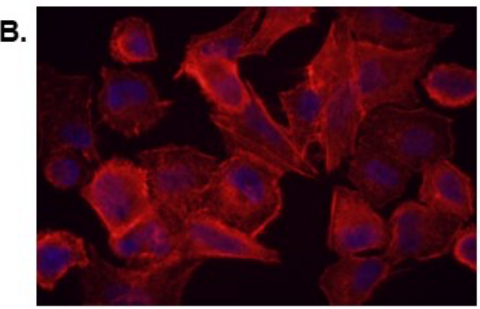

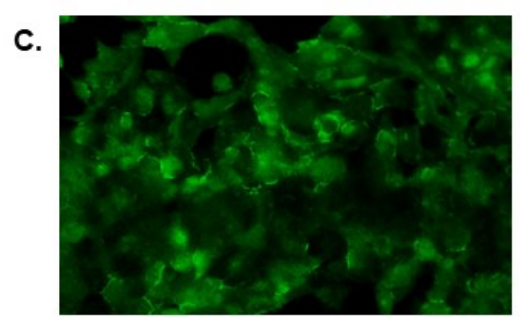

D.

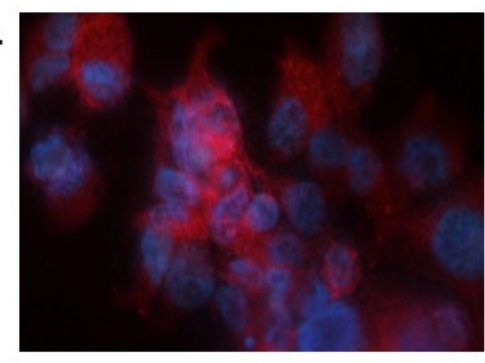

E.

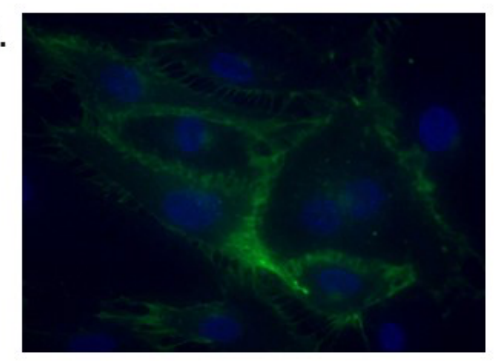

F.

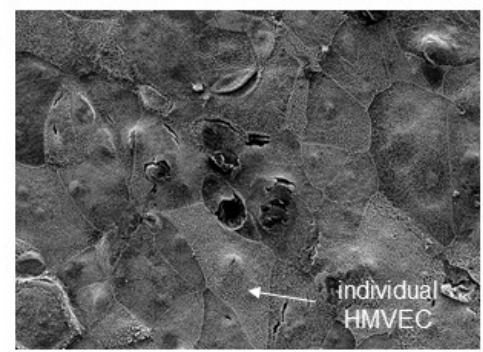

G.

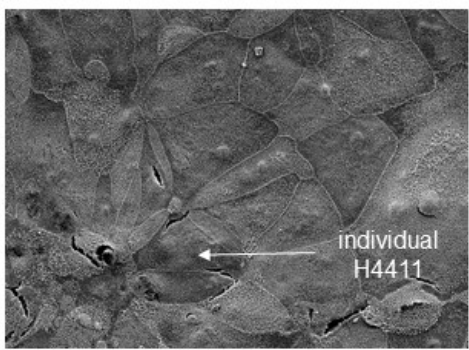

Figure 6. Cell adherence, sustained viability and cell specificity during co-culturing on thinned PSi membranes. (A and B) Fluorescence microscope images (400X magnification) of epithelial (H441) and endothelial (HMVEC) cells on the topside of the 25-micron PSi membrane on a fabricated annulus stained with phalloidin (red) and DAPI (blue). (C) Fluorescence microscope images (100X magnification) of viable H441 cells (Calcein-AM) seeded for 14 days on the topside of a PSi membrane within a fabricated annulus. (D and E) Fluorescence microscope images (1000X magnification) of H441 and HMVEC cells on a nano-PSi membrane stained with an epithelial-specific marker (Occludin, red) and endothelial-specific marker (VE cadherin, green) during a co-culture experiment. (F and G) SEM images (200X magnification) of HMVEC and H441 cells adhered to thinned, nano-PSi membranes following a co-culture experiment. H441 cells cultured on the bottom side of the nano-PSi membrane and HMVECs were cultured on the topside of the nano-PSi membrane.

Previous cell culture studies have shown that porous nanocrystalline silicon (pnc-Si) is thinned in cell culture media over 30 hours and that cells accelerate the onset of dissolution by altering the $\mathrm{pH}$ or through other chemical mechanisms [37]. Slight variations in the thicknesses and morphology of the PSi membranes were identified during the initial longer-term cell culture 
experiments, therefore, we wanted to determine if cellular or acellular effects altered NP and/or PSi over the 14-day time period. To this end, the thicknesses of thinned NP and PSi membranes were quantified via SEM analysis prior to cell culture and after 14 days in either cell culture media alone, cultured with adherent HMVECs, or cultured with THP-1 macrophages. For these experiments, all cell types were adhered to one side of the silicon structures. Membranes were trypsinized and treated extensively with solvents (methanol, isopropanol, and acetone) to remove all adherent cells and any cell-associated secreted components. NP and PSi membrane thicknesses did not change after 14 days in cell culture alone or cultured with adherent HMVECs (Figure 7 A). However, over the course of 14 days, PSi membranes but not NP wafers underwent a significant reduction in thickness of approximately $25 \%$ in the presence of adherent macrophages (Figure 7 A). To determine a possible cellular mechanism of PSi thinning, the membranes were fixed and analyzed through SEM without prior trypsinization to maintain adherent macrophages on the membranes. THP-1 macrophages were observed trafficking through the PSi membranes (Figure $7 \mathrm{~B}$ and C). This was not a single chance event since macrophages adjacent to each other were identified on the same PSi membrane trafficking through simultaneously (Figure 7 B). Upon closer observation, adherent macrophages significantly altered the PSi membranes and increased pore diameters, which were initially within the nanometer range (day 0 ) created by the anodization process, into micron-scale pores (greater than $10 \mu \mathrm{m}$ diameter) through a cellular or mechanical process (Figure 7 B-E). Higher magnification images identified pseudopodia (Figure 7 D) being created by the macrophages within the PSi and some macrophages that nearly trafficked to the opposing side of the membrane with the periphery of their plasma membranes (Figure $7 \mathrm{E}$ ). These data indicate cellular process enhanced the biodegradation of PSi membranes in vitro. 


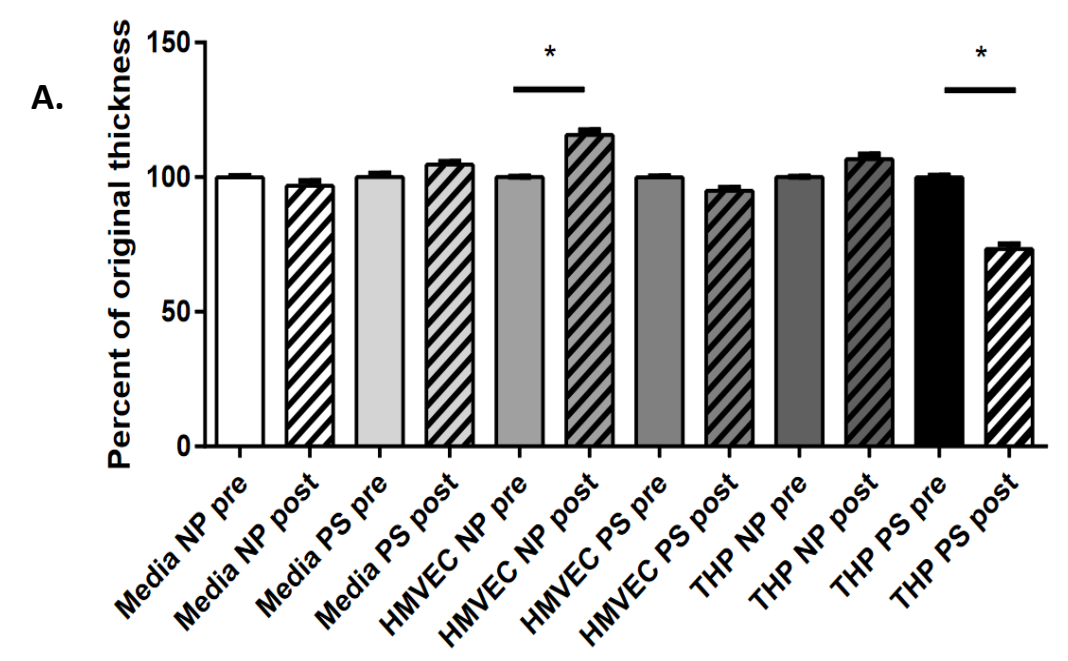

B.

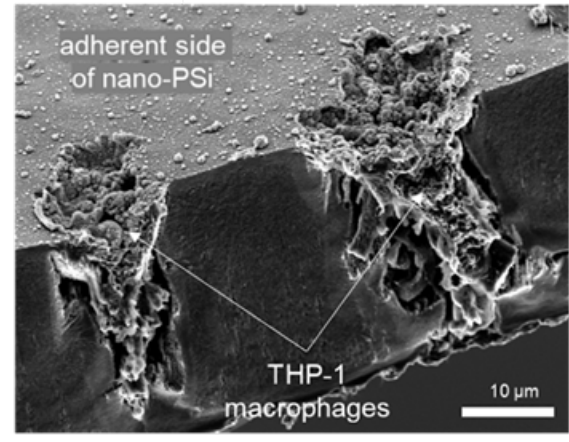

D.

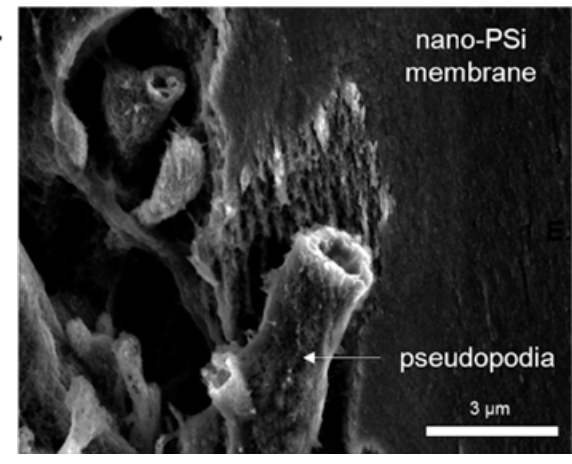

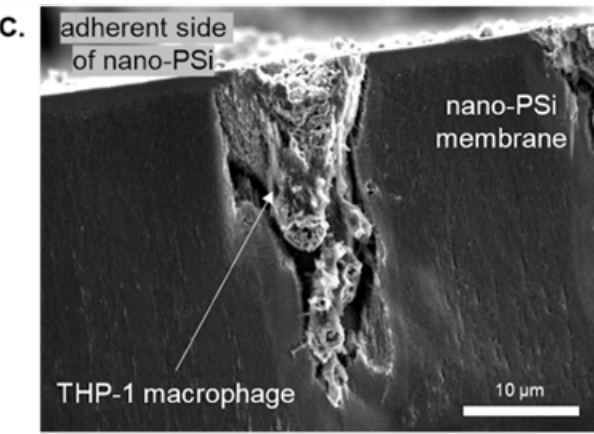

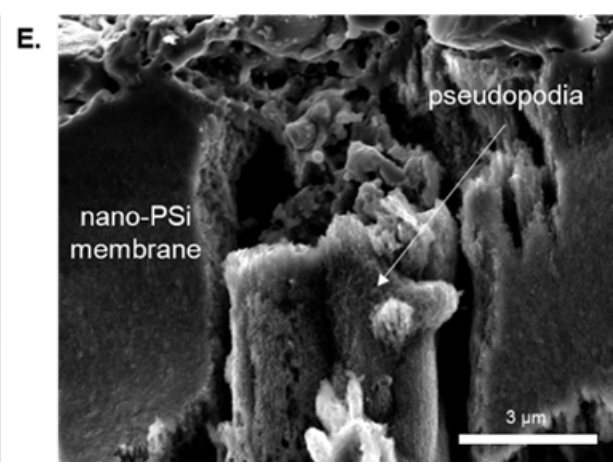

Figure 7: Silicon dissolution and observed trafficking of human macrophages through thinned, nano-PSi membranes via SEM analysis. (A) Human macrophages (THP-1 cells) and endothelial cells (HMVECs) were adhered to thinned, nano-PSi membranes and cultured at $37^{\circ} \mathrm{C}$ in cell culture media. After fourteen days, the membranes and cells were fixed and stained for SEM analysis. Cross-section distances were quantified, and the percent dissolution of the silicon membranes was quantified in triplicate $(n=3)$. Data presented are mean \pm SD. Asterisks represent a significant difference between membrane thicknesses before and after cell culture. $(\mathrm{p}<0.05)$. The data represent the aggregate of three biological replicates of the same 14-day procedure. (B and C) Representative SEM image of a human macrophage altering the silicon membrane through mechanical or chemical mechanisms imaged at 2,000x and 2,500x respectively. (D and E) Higher magnification of pseudopodia and the membrane periphery of a human macrophage transforming the nano-PSi membrane imaged at 8,000x and 9,500x respectively. 
While these experiments were conducted within the fabricated annuli (Figure 5) to evaluate cellular interactions and potential use of PSi membranes in organ-on-chip technology, we ultimately aim to use the fabricated PSi membranes in more complex microfluidic organ-on-chip devices. As a proof of concept, we developed a preliminary microfluidic LOAC device with independent channels that are unified by a center culture well with a PSi membrane in between (Figure S3). This preliminary design mimics conventional organ-on-chip devices where independent channels can be seeded with varying cell types separated by a membrane that serves to recapitulate the interstitial space in order to study tissue interfaces. Due to the reproducibility associated with fabricating tissue culture annuli containing PSi membranes, we chose to continue to use the techniques previously described that were used to bond PDMS to PSi membranes. While this method proved effective, this was done with large cell culture wells that were not connected to microfluid channels. In order to create cell culture wells that could be connected to microfluidic channels within PDMS, we utilized SU-8 soft lithography to generate negative molds. These negative molds were designed so that culture wells could be patterned into PDMS, layered, and bonded in such a way that both epithelial and endothelial cell types could be cultured within their respective regions separated by a PSi membrane (Figure S3). In addition, the independent microfluidic channels were connected to tubing so that they may be combined with a fluid system that would allow for the continuous supply of nutrient media and more closely mimic the parenchymal and vascular compartments of the lung at the blood-gas exchange interface. In future studies, we hope to use this design, or similar designs, to carry out detailed studies with the intention of further understanding pulmonary disease pathology and drug toxicity.

\subsection{Implications of the novel porous silicon membrane}

Our developed methods allow for a novel fabrication of biocompatible thinned PSi membranes. While thinning silicon wafers using $\mathrm{KOH}$ and anodization are well-established techniques in the field, our work presents a different way of combining these techniques in order to generate viable, reproducible membranes for use in organ-on-chip technology. In our design, silicon wafers can be readily loaded into PTFE etch rings and submerged in a solution of $\mathrm{KOH}$, and accurately and uniformly be etched to desired thicknesses. As previously described by Wang et al. [33], there is an observable increase in light transmission through the membrane as the silicon wafers become increasingly thin which was further evidence that the wafers were being thinned to the targeted 
thicknesses. Our developed method for anodization is unique because rather than using conventional "lift-off" methods [43], we utilize a PTFE dual tank etch cell to obtain large area ultra-thin fully PSi membranes that are mechanically stable and can be readily integrated into PDMS organ-on-chip devices. As previously discussed, we have the capabilities to generate PSi membranes with varying pore sizes ( $\sim 15.5 \mathrm{~nm}$ to $0.5 \mu \mathrm{m}$ in diameter), porosity, and thicknesses. While outside the scope of this work, i.e., a proof-of-concept study, ongoing experiments are focused on the evaluation of the formation parameter space of PSi membranes and their associated properties (mechanical, biocompatibility, biodegradability, chemical stability) relative to application in organs-on-a-chip.

The thickness, pore sizes and porosity of the fabricated PSi membranes described herein are adjustable (i.e., tunable) and can be compared to previous studies utilizing different materials and anodizing parameters. For example, porous nanocrystalline silicon (pnc-Si) membranes are significantly thinner (10 to $50 \mathrm{~nm}$ ) and have pore sizes that are only 3 to $80 \mathrm{~nm}$ in diameter and exhibit a low level of porosity (15\%) compared to the PSi membranes described in this study (50 - 76\%) [37]. Track-etched membranes made from either polycarbonate (PC), poly(ethylene terephalate) (PET) or polyester-sulfone (PES) also exhibit low pore densities and are limited in terms of pore sizes $(450 \mathrm{~nm})$ compared to this study [44]. However, improved isoporous membranes made from polyester have been reported with ten times greater porosity with pore sizes ranging from 0.7 to 50 microns. Finally, other membrane separation copolymer materials, such as gelatin methacrylate (GelMA) and dextran glycidyl methacrylate (DexMA) have also been shown to be tunable and have pore sizes ranging from 20 to 35 microns similar to the PSi membranes described above [45].

Based on previously published literature [58], we sought to utilize a high current generator to electrochemically bond the fabricated novel PSi membranes into PDMS devices that were engineered to evaluate the biocompatibility, biodegradability, and overall potential of PSi membranes in organ-on-chip technology. Using this model, cell adherence and viability were observed and characterized in order to inform us about the potential of this membrane in an organon-chip device.

Our data indicate that PSi provides an adherent, biocompatible surface for multiple cell types (epithelial, endothelial and macrophage cells) that are viable over a two-week period in cell culture. 
We observed increased cell adhesion onto PSi, which had increased surface roughness compared to bulk silicon, possibly due to increased surface area or improved receptor interactions as seen in previous studies [52]. Improved adhesion to macro- and nano-PSi substrates has been previously reported using human aortic endothelial cells, which utilized small cell protrusions (pseudopodia) to adhere to the macroporous surface [51]. Psuedopodia were observed on nano-PSi membranes via SEM.

The vast majority of fabricated organ-on-a-chip devices use difficult-to-manufacture thin PDMS membranes $(\sim 10-25 \mu \mathrm{m})$ to create structural support between cell types [56]. One significant difference in this study compared to previous PDMS organ-on-a-chip devices is that macrophages, epithelial, and endothelial cells adhered directly to the PSi membrane. PDMS membranes require both oxygen plasma treatment (to reduce hydrophobicity) and different extracellular matrix (ECM) coatings (to enhance cell attachment) for proper cell seeding that have transient effects in culture [56-58]. The PSi membranes fabricated in this paper did not require any additional treatments prior to cell culture and no issues were observed with cell adhesion for up to 14 days. Therefore, PSi membranes may be an alternative to current PDMS membranes. Compared to the hydrophilic and biocompatible nature of PSi, the hydrophobicity of PDMS may lead to uncrosslinked PDMS leaching from the surface into cell culture media and may absorb exogenous hydrophobic molecules that may lead to potential problems during longer-term, chronic studies [28,30]. In addition, future studies with PSi membranes may enable us to determine the extent to which cells can secrete and create de novo ECM components possibly with the addition of pulmonary fibroblasts.

During long-term culture, PSi was further thinned and penetrated by human macrophages through an unknown biomechanical or biochemical mechanism. The remarkable dissolution of the PSi surface observed in this study is in agreement with previous reports that indicate PSi and porous nanocrystalline silicon can be dissolved in aqueous solutions ( $\mathrm{pH}$ of 7 and $37^{\circ} \mathrm{C}$ ) over several days [36]. Degradation of PSi has also been shown to be independent of material size, occurs in the presence of cell culture components (FBS), occurs more quickly with unmodified PSi, and its degradation products (silicic acid and orthosilicic acid) are non-toxic [59]. Indeed, PSi has been used in nanomedicine therapeutic studies utilizing PSi nanoparticles, because they are dissolvable structures that are biodegradable [60]. Cell attachment and movement through PSi of 
undifferentiated THP-1 monocytes were evident during long-term culture (Fig. 7). THP-1 monocytes are sensitive to culture conditions that can alter THP-1 morphology as well as affect their response to differentiation stimuli [61]. The adherence of the undifferentiated macrophages was unexpected because these cells had not been exposed to PMA, which differentiates suspension THP-1 monocytes into mitotically inactive macrophages [54]. Adherence to PSi may occur through specific receptors, such as scavenger receptors that recognize specific repeating patterns, and binding may provide intracellular signals that determine cell direction via actin polymerization or important differentiation signaling [61]. Cellular forces for chemotaxis and adhesion have been quantified in multiple studies and occur between 10 and 7000 piconewtons [62]. Therefore, future studies will determine the extent to which biomechanical forces and biochemical pathways alter PSi during long-term culture.

\section{Conclusion}

In summary, we report on a novel method to fabricate a viable, reproducible PSi membrane that can be successfully integrated into PDMS-based organ-on-chip systems. This fabrication approach yields flexibility in setting the morphological and structural characteristics of the thin PSi membranes, such as pore diameter, porosity, and membrane thickness. Our study suggests that our PSi membranes may provide an improved alternative tissue interface, as opposed to conventional PDMS membranes used in current organ-on-chip technologies, because PSi membranes enable direct cell adhesion to both sides of the PSi membrane and the PSi membrane biodegrades over time. These advantages are due to the fact that cells do not readily remodel PDMS membranes compared to dynamic organ systems where cells interact with extracellular interfaces and traffic through tissue layers (i.e., immunological responses). Our model facilitates such interactions and may in the future more accurately recapitulate these dynamic in vivo systems. The use of these fabricated PSi membranes in organ-on-chip technology may allow for more accurate assessments of potential therapeutics and further our understanding of human disease pathology. Future studies will seek to understand and characterize multiple unique properties of the proposed PSi membranes. Furthermore, we will also evaluate the efficacy of PSi membranes in complex microfluidic organ-on-chip devices. Lastly, we will refine strategies to produce membranes that mimic the anatomical distance $(\sim 1 \mu \mathrm{m})$ between cellular populations at the alveolocapillary barrier. 


\section{Conflicts of interest}

The authors declare no conflicts of interest.

\section{Acknowledgments}

We would like to thank the faculty in the Fort Lewis College Department of Physics \& Engineering, Department of Biology, and Department of Chemistry \& Biochemistry for their generosity and expertise throughout this project. The success of this work was made possible with guidance and expertise provided by Fort Lewis College machinists Gabriel Sylvester and Jason Wagner. We would also like to thank Lily Vonesh and Avery Killifer for their aid in the laboratory as well as Michael Paffett Ph.D. for his microscopy skills. This work would not have been possible without the support of project coordinators Jaimee McCullough, Marnie Clay, Nicole Carey, and Steven Fenster. Graphical abstract and Figure 1 were created using BioRender.com. This project was graciously funded by the NSF PREM for Functional Nanomaterials [Award \#1827847], the NIH MARC U*STAR [Award \#T34GM092711], STROBE NSF Science and Technology Center on Real-Time Functional Imaging [Award \#1548924], by the National Institute Of General Medical Sciences of the National Institutes of Health under Award Number SC3GM141838 and by the UNM Comprehensive Cancer Center Support Grant NCI P30CA118100 using the Fluorescence Microscopy and Cell Imaging shared resource.

\section{References}

[1] D.E. Ingber, Reverse Engineering Human Pathophysiology with Organs-on-Chips, Cell. 164 (2016) 1105-1109. https://doi.org/10.1016/j.cell.2016.02.049.

[2] D.G. Hackam, D.A. Redelmeier, Translation of Research Evidence From Animals to Humans, JAMA. 296 (2006) 1727-1732. https://doi.org/10.1001/jama.296.14.1731.

[3] Y. Buhidma, K. Rukavina, K.R. Chaudhuri, S. Duty, Potential of animal models for advancing the understanding and treatment of pain in Parkinson's disease, Npj Park. Dis. 6 (2020) 1-7. https://doi.org/10.1038/s41531-019-0104-6.

[4] M. Van Den Buuse, B. Garner, A. Gogos, S. Kusljic, Importance of animal models in schizophrenia research, (2005).

[5] J.G. Neyt, J.A. Buckwalter, N.C. Carroll, Use of animal models in musculoskeletal 
research, Iowa Orthop. J. 18 (1998) 118-123.

[6] A.I. Pearce, R.G. Richards, S. Milz, E. Schneider, S.G. Pearce, Animal models for implant biomaterial research in bone: A review, Eur. Cells Mater. 13 (2007) 1-10. https://doi.org/10.22203/eCM.v013a01.

[7] G. Dranoff, Experimental mouse tumour models: What can be learnt about human cancer immunology?, Nat. Rev. Immunol. 12 (2012) 61-66. https://doi.org/10.1038/nri3129.

[8] C.H. Wong, K.W. Siah, A.W. Lo, Estimation of clinical trial success rates and related parameters, Biostatistics. 20 (2019) 273-286. https://doi.org/10.1093/biostatistics/kxx069.

[9] O.A. Ali, E. Doherty, W.J. Bell, T. Fradet, J. Hudak, M.T. Laliberte, D.J. Mooney, D.F. Emerich, Biomaterial-based vaccine induces regression of established intracranial glioma in rats, Pharm. Res. 28 (2011) 1074-1080. https://doi.org/10.1007/s11095-010-0361-x.

[10] D.W. Infanger, M.E. Lynch, C. Fischbach, Engineered Culture Models for Studies of Tumor-Microenvironment Interactions, Annu. Rev. Biomed. Eng. 15 (2013) 29-53. https://doi.org/10.1146/annurev-bioeng-071811-150028.

[11] H.H.G. Song, K.M. Park, S. Gerecht, Hydrogels to model 3D in vitro microenvironment of tumor vascularization, Adv. Drug Deliv. Rev. 79 (2014) 19-29. https://doi.org/10.1016/j.addr.2014.06.002.

[12] D. Huh, Y.S. Torisawa, G.A. Hamilton, H.J. Kim, D.E. Ingber, P. Formentín, Ú. Catalán, L. Pol, S. Fernández-Castillejo, R. Solà, L.F. Marsal, S.E. Dunsmore, D.E. Rannels, E.R. Weibel, B. Zhou, X. Gao, C. Wang, Z. Ye, Y. Gao, J. Xie, X. Wu, W. Wen, P. Formentín, M. Alba, U. Catalán, S. Fernández-Castillejo, J. Pallarès, R. Solà, L.F. Marsal, S.P. Low, K.A. Williams, L.T. Canham, N.H. Voelcker, J.G. Croissant, Y. Fatieiev, N.M. Khashab, E.J. Yun, W. Lorizio, G. Seedorf, S.H. Abman, T.H. Vu, T.R. Gaborski, J.L. Snyder, C.C. Striemer, D.Z. Fang, M. Hoffman, P.M. Fauchet, J.L. McGrath, I.Y. Adamson, L. Young, H.H. Chung, M. Mireles, B.J. Kwarta, T.R. Gaborski, G. Burgstaller, B. Oehrle, M. Gerckens, E.S. White, H.B. Schiller, O. Eickelberg, M.I. Hermanns, R.E. Unger, K. Kehe, K. Peters, C.J. Kirkpatrick, A. Mossu, M. Rosito, T. Khire, H. Li Chung, H. Nishihara, I. Gruber, E. Luke, L. Dehouck, F. Sallusto, F. Gosselet, J.L. McGrath, B. Engelhardt, J.H. 
Park, L. Gu, G. von Maltzahn, E. Ruoslahti, S.N. Bhatia, M.J. Sailor, M.W. Toepke, D.J. Beebe, S.H. Ma, L.A. Lepak, R.J. Hussain, W. Shain, M.L. Shuler, D. Huh, H.J. Kim, J.P. Fraser, D.E. Shea, M. Khan, A. Bahinski, G.A. Hamilton, D.E. Ingber, B.H. Gu, M.C. Madison, D. Corry, F. Kheradmand, D. Huh, G.A. Hamilton, D.E. Ingber, B.J. Gill, J.L. West, K.H. Benam, R. Novak, T.C. Ferrante, Y. Choe, D.E. Ingber, J.J. Miller, R.N. Carter, K.B. McNabb, J.-P.S. DesOrmeaux, C.C. Striemer, J.D. Winans, T.R. Gaborski, D.E. Ingber, C.A. Prestidge, T.J. Barnes, C.H. Lau, C. Barnett, A. Loni, L.T. Canham, D. Huh, B.D. Matthews, A. Mammoto, M. Montoya-Zavala, H. Yuan Hsin, D.E. Ingber, K.H. Benam, R. Villenave, C. Lucchesi, A. Varone, C. Hubeau, H.H. Lee, S.E. Alves, M. Salmon, T.C. Ferrante, J.C. Weaver, A. Bahinski, G.A. Hamilton, D.E. Ingber, G.J. Chader, A silicon nanomembrane platform for the visualization of immune cell trafficking across the human blood-brain barrier under flow, Lab Chip. 8 (2018) 395-410. https://doi.org/10.1177/0271678X18820584.

[13] D.J. Beebe, D.E. Ingber, J. Den Toonder, Organs on Chips, Lab Chip. 13 (2013) 34473448. https://doi.org/10.1039/c3lc90080k.

[14] Fabre, K., Berridge, B., Proctor, W. R., Ralston, S., Will, Y., Baran, S. W., Yoder, G., \& Van Vleet, T. R. Introduction to a manuscript series on the characterization and use of microphysiological systems (MPS) in pharmaceutical safety and ADME applications. Lab Chip (2020) 20(6), 1049-1057. https://doi.org/10.1039/c9lc01168d

[15] S.N. Bhatia, D.E. Ingber, Microfluidic organs-on-chips, Nat Biotechnol. 32 (2014) 760772. https://doi.org/10.1038/nbt.2989.

[16] D. Huh, B.D. Matthews, A. Mammoto, M. Montoya-Zavala, H. Yuan Hsin, D.E. Ingber, Reconstituting organ-level lung functions on a chip, Science (80-. ). 328 (2010) 16621668. https://doi.org/10.1126/science.1188302.

[17] D. Huh, Y.S. Torisawa, G.A. Hamilton, H.J. Kim, D.E. Ingber, Microengineered physiological biomimicry: Organs-on-Chips, Lab Chip. 12 (2012) 2156-2164. https://doi.org/10.1039/c2lc40089h.

[18] C. Arrigoni, S. Lopa, C. Candrian, M. Moretti, Organs-on-a-chip as model systems for multifactorial musculoskeletal diseases, Curr. Opin. Biotechnol. 63 (2020) 79-88. 
https://doi.org/10.1016/j.copbio.2019.12.006.

[19] S. Hassan, S. Sebastian, S. Maharjan, A. Lesha, A. Carpenter, X. Liu, X. Xie, C. Livermore, Y.S. Zhang, A. Zarrinpar, Liver-on-a-Chip Models of Fatty Liver Disease, Hepatology. (2020). https://doi.org/10.1002/hep.31106.

[20] H.M. Jeon, K. Kim, K.C. Choi, G.Y. Sung, Side-effect test of sorafenib using 3-D skin equivalent based on microfluidic skin-on-a-chip, J. Ind. Eng. Chem. 82 (2020) 71-80. https://doi.org/10.1016/j.jiec.2019.09.044.

[21] M. Kasendra, A. Tovaglieri, A. Sontheimer-Phelps, S. Jalili-Firoozinezhad, A. Bein, A. Chalkiadaki, W. Scholl, C. Zhang, H. Rickner, C.A. Richmond, H. Li, D.T. Breault, D.E. Ingber, Development of a primary human Small Intestine-on-a-Chip using biopsy-derived organoids, Sci. Rep. 8 (2018) 1-14. https://doi.org/10.1038/s41598-018-21201-7.

[22] M.A.U. Khalid, Y.S. Kim, M. Ali, B.G. Lee, Y.J. Cho, K.H. Choi, A lung cancer-on-chip platform with integrated biosensors for physiological monitoring and toxicity assessment, Biochem. Eng. J. 155 (2020) 107469. https://doi.org/10.1016/j.bej.2019.107469.

[23] C. Poussin, 3D human microvessel-on-a-chip model for studying monocyte-toendothelium adhesion under flow - application in systems toxicology, ALTEX. 37 (2019) 47-63. https://doi.org/10.14573/altex.1811301.

[24] J. Vriend, J.G.P. Peters, T.T.G. Nieskens, R. Škovroňová, N. Blaimschein, M. Schmidts, R. Roepman, T.J.J. Schirris, F.G.M. Russel, R. Masereeuw, M.J. Wilmer, Flow stimulates drug transport in a human kidney proximal tubule-on-a-chip independent of primary cilia, Biochim. Biophys. Acta - Gen. Subj. 1864 (2020). https://doi.org/10.1016/j.bbagen.2019.129433.

[25] R.N. Carter, S.M. Casillo, A.R. Mazzocchi, J.S. DesOrmeaux, J.A. Roussie, T.R. Gaborski, Ultrathin transparent membranes for cellular barrier and co-culture models, Biofabrication. 9 (2017) 15019. https://doi.org/10.1088/1758-5090/aa5ba7.

[26] Haase, K., Kamm, R.D., Advances in on-chip vascularization, Regen Med (2017). 12(3), 285-302. https://doi.org/10.2217/rme-2016-0152

[27] Si, L., Bai, H., Rodas, M. et al. A human-airway-on-a-chip for the rapid identification of 
candidate antiviral therapeutics and prophylactics. Nat Biomed Eng (2021).

https://doi.org/10.1038/s41551-021-00718-9

[28] K.J. Regehr, M. Domenech, J.T. Koepsel, K.C. Carver, S.J. Ellison-Zelski, W.L. Murphy, L.A. Schuler, E.T. Alarid, D.J. Beebe, Biological implications of polydimethylsiloxanebased microfluidic cell culture, Lab Chip. 9 (2009) 2132-2139.

https://doi.org/10.1039/b903043c.

[29] J.D. Stucki, N. Hobi, A. Galimov, A.O. Stucki, N. Schneider-Daum, C.M. Lehr, H. Huwer, M. Frick, M. Funke-Chambour, T. Geiser, O.T. Guenat, Medium throughput breathing human primary cell alveolus-on-chip model, Sci Rep. 8 (2018) 14359. https://doi.org/10.1038/s41598-018-32523-x.

[30] Benam KH, Villenave R, Lucchesi C, Varone A, Hubeau C, Lee HH, Alves SE, Salmon M, Ferrante TC, Weaver JC, Bahinski A, Hamilton GA, Ingber DE. Small airway-on-achip enables analysis of human lung inflammation and drug responses in vitro. Nat Methods. 2016 Feb;13(2):151-7. doi: 10.1038/nmeth.3697. Epub 2015 Dec 21. PMID: 26689262.

[31] M.W. Toepke, D.J. Beebe, PDMS absorption of small molecules and consequences in microfluidic applications, Lab Chip. 6 (2006) 1484-1486. https://doi.org/10.1039/b612140c.

[32] J.G. Croissant, Y. Fatieiev, N.M. Khashab, Degradability and Clearance of Silicon, Organosilica, Silsesquioxane, Silica Mixed Oxide, and Mesoporous Silica Nanoparticles, Adv. Mater. 29 (2017). https://doi.org/10.1002/adma.201604634.

[33] E. Jastrzebska, A. Zuchowska, S. Flis, P. Sokolowska, M. Bulka, A. Dybko, Z. Brzozka, Biological characterization of the modified poly(dimethylsiloxane) surfaces based on cell attachment and toxicity assays, Biomicrofluidics. 12 (2018). https://doi.org/10.1063/1.5035176.

[34] Zamprogno, P., Wüthrich, S., Achenbach, S. et al. Second-generation lung-on-a-chip with an array of stretchable alveoli made with a biological membrane. Commun Biol. 168 (2021). https://doi.org/10.1038/s42003-021-01695-0 
[35] Rofaani, E., Peng, J., Wang, L., He, Y., Huang, B., \& Chen, Y. Fabrication of ultrathin artificial basement membrane for epithelial cell cultur (2020) Microelec Eng, 232, 111407. https://doi.org/10.1016/j.mee.2020.111407

[36] S. Wang, B.D. Weil, Y. Li, K.X. Wang, E. Garnett, S. Fan, Y. Cui, Large-area freestanding ultrathin single-crystal silicon as processable materials, Nano Lett. 13 (2013) 4393-4398. https://doi.org/10.1021/nl402230v.

[37] A.A. Agrawal, B.J. Nehilla, K. V Reisig, T.R. Gaborski, D.Z. Fang, C.C. Striemer, P.M. Fauchet, J.L. McGrath, Porous nanocrystalline silicon membranes as highly permeable and molecularly thin substrates for cell culture, Biomaterials. 31 (2010) 5408-5417. https://doi.org/10.1016/j.biomaterials.2010.03.041.

[38] L.A. Beardslee, J. Stolwijk, D.A. Khaladj, M. Trebak, J. Halman, K.Y. Torrejon, N. Niamsiri, M. Bergkvist, A sacrificial process for fabrication of biodegradable polymer membranes with submicron thickness, J. Biomed. Mater. Res. - Part B Appl. Biomater. 104 (2016) 1192-1201. https://doi.org/10.1002/jbm.b.33464.

[39] D.Z. Fang, C.C. Striemer, T.R. Gaborski, J.L. McGrath, P.M. Fauchet, Methods for controlling the pore properties of ultra-thin nanocrystalline silicon membranes, J Phys Condens Matter. 22 (2010) 454134. https://doi.org/10.1088/0953-8984/22/45/454134.

[40] S.H. Ma, L.A. Lepak, R.J. Hussain, W. Shain, M.L. Shuler, An endothelial and astrocyte co-culture model of the blood-brain barrier utilizing an ultra-thin, nanofabricated silicon nitride membrane, Lab Chip. 5 (2005) 74-85. https://doi.org/10.1039/b405713a.

[41] A. Mossu, M. Rosito, T. Khire, H. Li Chung, H. Nishihara, I. Gruber, E. Luke, L. Dehouck, F. Sallusto, F. Gosselet, J.L. McGrath, B. Engelhardt, A silicon nanomembrane platform for the visualization of immune cell trafficking across the human blood-brain barrier under flow, J. Cereb. Blood Flow Metab. 39 (2019) 395-410. https://doi.org/10.1177/0271678X18820584.

[42] R. Martín-Palma, M. Manso-Silván, V. Torres-Costa, Biomedical applications of nanostructured porous silicon: A review, J. Nanophotonics. 4 (2010). https://doi.org/10.1117/1.3496303. 
[43] J.J. Miller, R.N. Carter, K.B. McNabb, J.P.S. Desormeaux, C.C. Striemer, J.D. Winans, T.R. Gaborski, Lift-off of large-scale ultrathin nanomembranes, J. Micromechanics Microengineering. 25 (2015) 015011. https://doi.org/10.1088/0960-1317/25/1/015011.

[44] Rijn. (2004). Nano and micro engineered membrane technology. Amsterdam Boston: Elsevier.

[45] Wang H, Zhou L, Liao J, Tan Y, Ouyang K, Ning C, Ni G, Tan G. Cell-laden photocrosslinked GelMA-DexMA copolymer hydrogels with tunable mechanical properties for tissue engineering. J Mater Sci Mater Med. (2014) Sep;25(9):2173-83. doi: 10.1007/s10856-014-5261-x. Epub 2014 Jul 10. PMID: 25008369.

[46] Crapo, J.D., Barry, B.E., Gehr, P., Bachofen, M., Weibel, E.R., Cell number and cell characteristics of the normal human lung, Am Rev Respir Dis. 125 (1982) 740-745. https://doi.org/10.1164/arrd.1982.125.6.740.

[47] Weibel, E.R., Lung morphometry: the link between structure and function, Cell Tissue Res. 367 (2017) 413-426. https://doi.org/10.1007/s00441-016-2541-4.

[48] B.H. Chueh, D. Huh, C.R. Kyrtsos, T. Houssin, N. Futai, S. Takayama, Leakage-free bonding of porous membranes into layered microfluidic array systems, Anal. Chem. 79 (2007) 3504-3508. https://doi.org/10.1021/ac062118p.

[49] M.I. Hermanns, R.E. Unger, K. Kehe, K. Peters, C.J. Kirkpatrick, Lung epithelial cell lines in coculture with human pulmonary microvascular endothelial cells: Development of an alveolo-capillary barrier in vitro, Lab. Investig. (2004). https://doi.org/10.1038/labinvest.3700081.

[50] F. Akther, S.B. Yakob, N.T. Nguyen, H.T. Ta, Surface Modification Techniques for Endothelial Cell Seeding in PDMS Microfluidic Devices, Biosensors. 10 (2020). https://doi.org/10.3390/bios 10110182.

[51] P. Formentín, M. Alba, Ú. Catalán, S. Fernández-Castillejo, J. Pallarès, R. Solà, L.F. Marsal, Effects of macro- versus nanoporous silicon substrates on human aortic endothelial cell behavior, Nanoscale Res. Lett. 9 (2014) 421. https://doi.org/10.1186/1556-276X-9-421. 
[52] M. Uhlén, L. Fagerberg, B.M. Hallström, C. Lindskog, P. Oksvold, A. Mardinoglu, Å. Sivertsson, C. Kampf, E. Sjöstedt, A. Asplund, I. Olsson, K. Edlund, E. Lundberg, S. Navani, C.A. Szigyarto, J. Odeberg, D. Djureinovic, J.O. Takanen, S. Hober, T. Alm, P.H. Edqvist, H. Berling, H. Tegel, J. Mulder, J. Rockberg, P. Nilsson, J.M. Schwenk, M. Hamsten, K. von Feilitzen, M. Forsberg, L. Persson, F. Johansson, M. Zwahlen, G. von Heijne, J. Nielsen, F. Pontén, Proteomics. Tissue-based map of the human proteome, Science (80-. ). 347 (2015) 1260419. https://doi.org/10.1126/science.1260419.

[53] R.D. Das, C. RoyChaudhuri, S. Maji, S. Das, H. Saha, Macroporous silicon based simple and efficient trapping platform for electrical detection of Salmonella typhimurium pathogens, Biosens Bioelectron. 24 (2009) 3215-3222. https://doi.org/10.1016/j.bios.2009.04.014.

[54] H. Schwende, E. Fitzke, P. Ambs, P. Dieter, Differences in the state of differentiation of THP-1 cells induced by phorbol ester and 1,25-dihydroxyvitamin D3, J. Leukoc. Biol. 59 (1996) 555-561.

https://fortlewis.idm.oclc.org/login?url=https://search.ebscohost.com/login.aspx?direct=tr $\mathrm{ue} \& \mathrm{db}=\mathrm{cmedm} \& \mathrm{AN}=8613704 \&$ site $=$ eds-live $\&$ scope $=$ site.

[55] S. Halldorsson, E. Lucumi, R. Gómez-Sjöberg, R.M.T. Fleming, Advantages and challenges of microfluidic cell culture in polydimethylsiloxane devices, Biosens Bioelectron. 63 (2015) 218-231. https://doi.org/10.1016/j.bios.2014.07.029.

[56] T. Fujii, PDMS-based microfluidic devices for biomedical applications, Microelectron. Eng. 61-62 (2002) 907-914. https://doi.org/https://doi.org/10.1016/S01679317(02)00494-X.

[57] S.H.C. Anderson, H. Elliott, D.J. Wallis, L.T. Canham, J.J. Powell, Dissolution of different forms of partially porous silicon wafers under simulated physiological conditions, Phys. Status Solidi. 197 (2003) 331-335.

[58] H. Hillborg, U.W. Gedde, Hydrophobicity recovery of polydimethylsiloxane after exposure to corona discharges, Polymer (Guildf). 39 (1998) 1991-1998.

[59] C.A. Prestidge, T.J. Barnes, C.H. Lau, C. Barnett, A. Loni, L. Canham, Mesoporous 
silicon: A platform for the delivery of therapeutics, Expert Opin. Drug Deliv. 4 (2007) 101-110. https://doi.org/10.1517/17425247.4.2.101.

[60] J.H. Park, L. Gu, G. Von Maltzahn, E. Ruoslahti, S.N. Bhatia, M.J. Sailor, Biodegradable luminescent porous silicon nanoparticles for in vivo applications, Nat. Mater. 8 (2009) 331-336. https://doi.org/10.1038/nmat2398.

[61] M.A. Forrester, H.J. Wassall, L.S. Hall, H. Cao, H.M. Wilson, R.N. Barker, M.A. Vickers, Similarities and differences in surface receptor expression by THP-1 monocytes and differentiated macrophages polarized using seven different conditioning regimens, Cell Immunol. 332 (2018) 58-76. https://doi.org/10.1016/j.cellimm.2018.07.008.

[62] R. Ananthakrishnan, A. Ehrlicher, The forces behind cell movement, Int J Biol Sci. 3 (2007) 303-317. https://doi.org/10.7150/ijbs.3.303. 


\section{Supplementary Information}
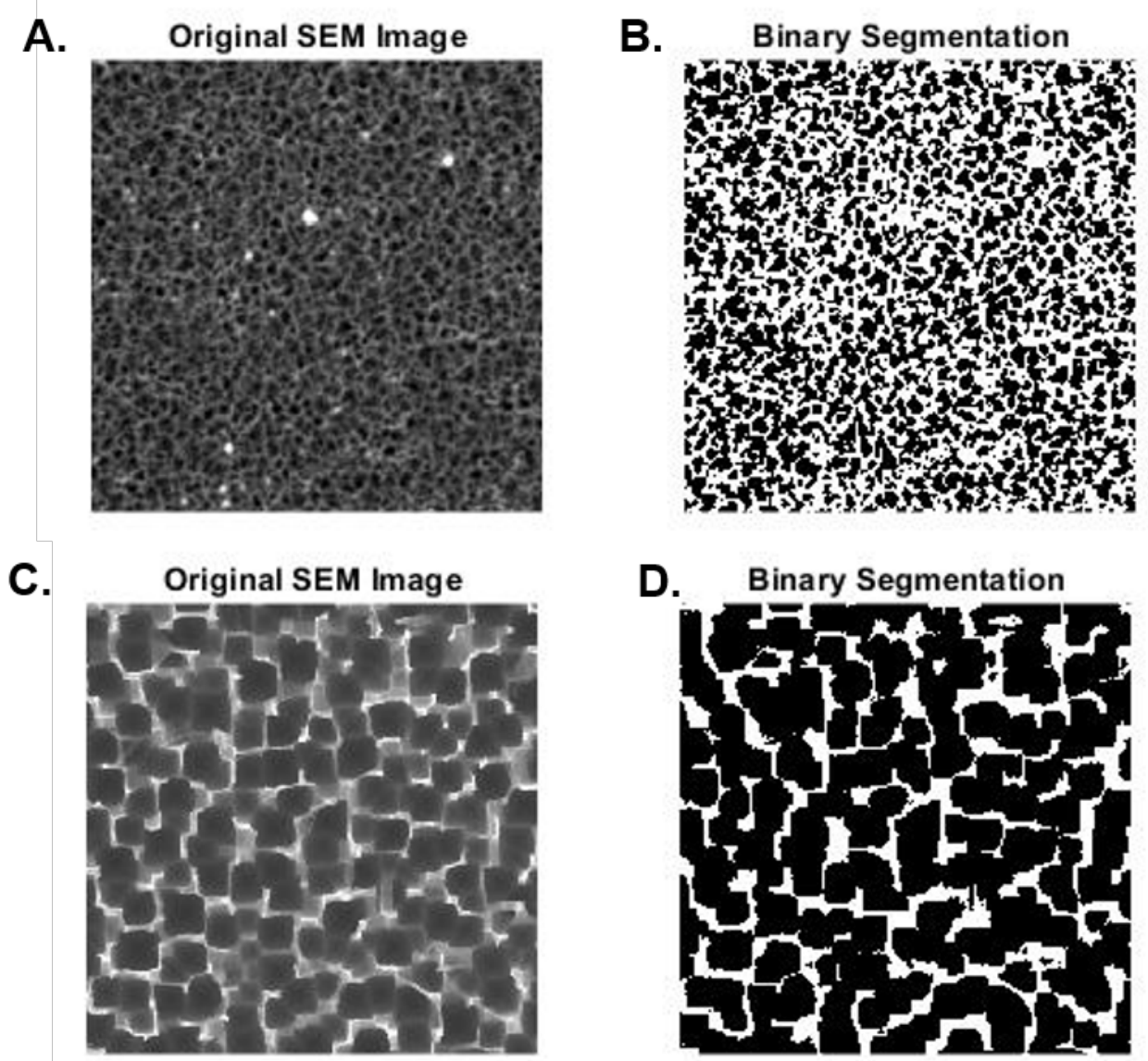

Figure S1. MATLAB image processing converting SEM images to grayscale, allowing percent porosity to be calculated. (A) Example SEM top-down image of top nano-PSi membrane used in image processing. (B) Image processing calculating an average percent porosity of 50.722\% porous and an average pore diameter of $15.5 \mathrm{~nm} \pm 6.21 \mathrm{~nm}$ for nano-PSi membranes. (C) Example SEM top-down image of top macro-PSi membrane used in image processing. (D) Image processing calculating an average percent porosity of $76.843 \%$ porous and an average pore diameter of $0.469 \mu \mathrm{m} \pm 0.210 \mu \mathrm{m}$ for macro-PSi membranes. 
A.
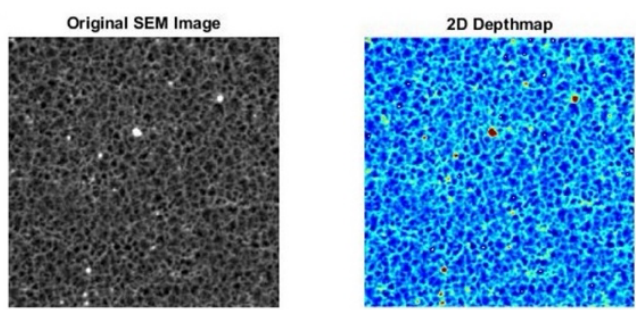

B.

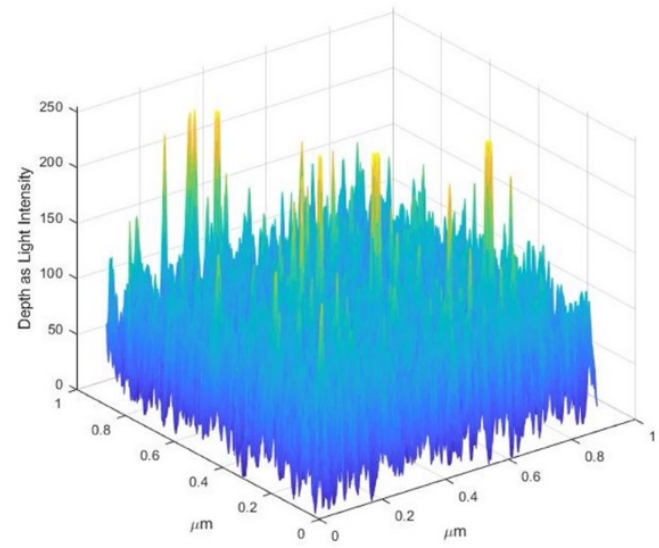

c.

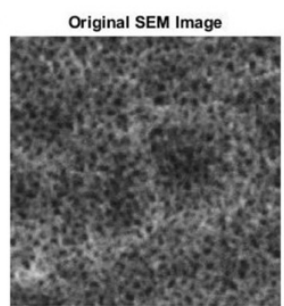

2D Depthmap

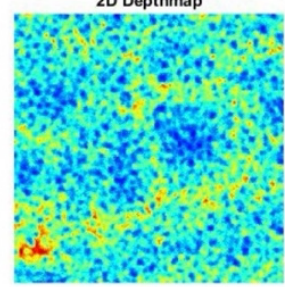

D.

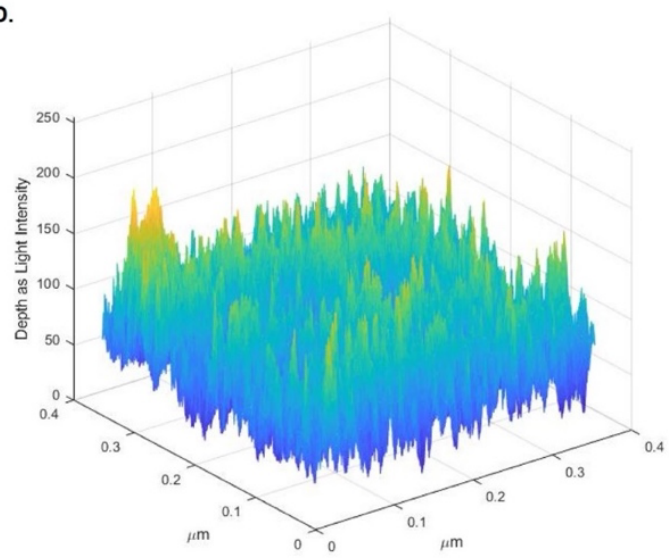

Figure S2. MATLAB image processing analyzing light intensity in SEM allowing for computed surface topography depth maps to be generated. (A) Image processing of the top side of a throughetched wafer used to generate a 2D depthmap. (B) 3D depthmap of the top side of a through-etched wafer generated using 2D depthmap image processing. (C) Image processing of backside of a through-etched wafer used to generate a 2D depthmap. (D) 3D depthmap of backside of a throughetched wafer generated using $2 \mathrm{D}$ depthmap image processing. 

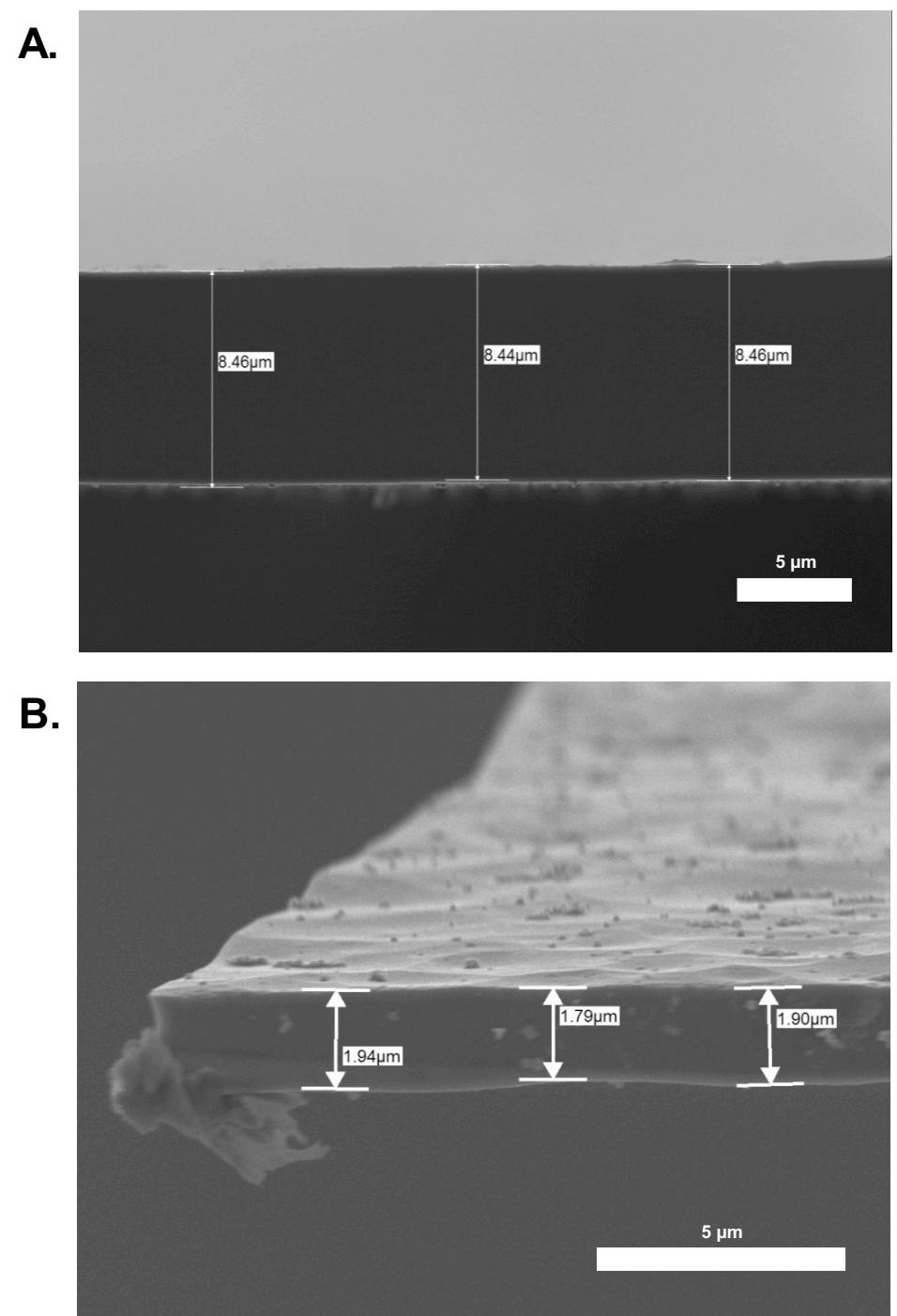

Figure S3. A prototype microfluidic LOAC device with a PSi membrane that would allow for independent seeding of both endothelial and epithelial pulmonary cells within their respective regions. Dimensions are noted in Materials and Methods 2.6. (A) The fabricated PDMS microfluidic device with independent channels visualized in blue and red. (B) Schematic of the LOAC microfluidic design in an exploded view detailing the center culture well and PSi membrane placement. (C) Schematic of LOAC microfluidic device from the top-down view detailing connection points for the implementation of a fluid system. 